\title{
The Poisson embedding approach to the Calderón problem
}

\author{
Matti Lassas $^{1} \cdot$ Tony Liimatainen $^{1}$ (]) $\cdot$ Mikko Salo $^{2}$ (I) \\ Received: 18 June 2018 / Revised: 17 December 2018 / Published online: 21 March 2019 \\ (c) The Author(s) 2019
}

\begin{abstract}
We introduce a new approach to the anisotropic Calderón problem, based on a map called Poisson embedding that identifies the points of a Riemannian manifold with distributions on its boundary. We give a new uniqueness result for a large class of Calderón type inverse problems for quasilinear equations in the real analytic case. The approach also leads to a new proof of the result of Lassas et al. (Annales de l' ENS 34(5):771-787, 2001) solving the Calderón problem on real analytic Riemannian manifolds. The proof uses the Poisson embedding to determine the harmonic functions in the manifold up to a harmonic morphism. The method also involves various Runge approximation results for linear elliptic equations.
\end{abstract}

\section{Introduction}

The anisotropic Calderón problem consists in determining a conductivity matrix of a medium, up to a change of coordinates fixing the boundary, from electrical voltage and current measurements on the boundary. In dimensions $n \geq 3$ this problem may be written geometrically as the determination of a Riemannian metric on a compact manifold with boundary from Dirichlet and Neumann data of harmonic functions. More precisely, if $(M, g)$ is a compact oriented Riemannian manifold with smooth boundary, we consider the Dirichlet problem for the Laplace-Beltrami operator $\Delta_{g}$,

\footnotetext{
Communicated by Y. Giga.

$凶$ Tony Liimatainen

tony.liimatainen@helsinki.fi

Matti Lassas

matti.lassas@helsinki.fi

Mikko Salo

mikko.j.salo@jyu.fi

1 Department of Mathematics and Statistics, University of Helsinki, Helsinki, Finland

2 Department of Mathematics and Statistics, University of Jyvaskyla, Jyvaskyla, Finland
} 


$$
\Delta_{g} u=0 \text { in } M,\left.\quad u\right|_{\partial M}=f
$$

and denote $u=u_{f}$. We define the Dirichlet-to-Neumann map (DN map)

$$
\Lambda_{g}: C^{\infty}(\partial M) \rightarrow C^{\infty}(\partial M), \quad \Lambda_{g} f=\left.\partial_{\nu} u_{f}\right|_{\partial M}
$$

where $\partial_{v}$ is the normal derivative on $\partial M$. One has the coordinate invariance

$$
\Lambda_{g}=\Lambda_{\phi^{*} g}
$$

for any diffeomorphism $\phi: M \rightarrow M$ fixing the boundary.

It is a long-standing conjecture [30] that if two Riemannian manifolds $\left(M_{1}, g_{1}\right)$ and $\left(M_{2}, g_{2}\right)$ with mutual boundary have the same DN maps, then there is a boundary fixing isometry between the manifolds. In this paper we give a candidate for this isometry:

The points $x_{1} \in M_{1}$ and $x_{2} \in M_{2}$ are to be identified if and only if for every $f \in C^{\infty}(\partial M)$ the harmonic extensions $u_{f}^{1}$ and $u_{f}^{2}$ of $f$ to $\left(M_{1}, g_{1}\right)$ and $\left(M_{2}, g_{2}\right)$ satisfy

$$
u_{f}^{1}\left(x_{1}\right)=u_{f}^{2}\left(x_{2}\right)
$$

For general Riemannian manifolds such an identification does not exist. However, if such an identification exists, we show that it induces a mapping $M_{1} \rightarrow M_{2}$, and that this mapping is the boundary fixing isometry required for the solution of the Calderón problem. The Calderón problem thus reduces to showing that the equality of DN maps implies that the above identification exists. In this case we say that the manifolds can be identified by their harmonic functions.

We introduce a tool for studying the existence of this identification. This will be an embedding $P$ of a Riemannian manifold into the linear dual of the space of smooth functions on its boundary. If $(M, g)$ is a compact Riemannian manifold with $C^{\infty}$ boundary and $x \in M$, then the value of $P$ at $x$ is a linear functional given by the formula

$$
P(x) f=u_{f}(x),
$$

where $u_{f} \in C^{\infty}(M)$ solves the Dirichlet problem

$$
\begin{aligned}
\Delta_{g} u_{f} & =0 \text { in } M, \\
u_{f} & =f \text { on } \partial M .
\end{aligned}
$$

We call the mapping $P$ the Poisson embedding, and it will be the main object of study of this paper. If $P_{1}\left(M_{1}\right) \subset P_{2}\left(M_{2}\right)$, then

$$
P_{2}^{-1} \circ P_{1}
$$


is a well-defined map $M_{1} \rightarrow M_{2}$, and we prove that it is a boundary fixing isometry if $n \geq 3$. If $n=2$, it is a conformal mapping. The condition $P_{1}\left(M_{1}\right) \subset P_{2}\left(M_{2}\right)$ is equivalent to existence of the identification (1).

There are different points of view to the Poisson embedding:

1. The Poisson formula for solutions of the Dirichlet problem gives that

$$
P(x) f=\int_{\partial M} \partial_{\nu_{y}} G(x, y) f(y) d S(y)
$$

where $G(x, y)$ is the Green function for $\Delta_{g}$ in $M$ and $\partial_{v_{y}} G(x, \cdot)$ is the Poisson kernel. Thus $P$ identifies the point $x$ in $M$ with the Poisson kernel $\partial_{v_{y}} G(x, \cdot)$ on $\partial M$ (hence the name Poisson embedding).

2. One also has the formula

$$
P(x) f=\int_{\partial M} f d \omega^{x}
$$

where $\omega^{x}$ is the harmonic measure for $\Delta_{g}$ at $x$. Thus $P$ identifies points in $M$ with measures on $\partial M$; points of $\partial M$ are identified with the corresponding Dirac measures, and points in $M^{\text {int }}$ are identified with $C^{\infty}$ functions since $d \omega^{x}=\partial_{\nu_{y}} G(x, \cdot) d S$ in this case.

3. For $x \in M^{\text {int }}$ one has $G(x, \cdot)=0$ on $\partial M$, and thus the knowledge of $\left.\partial_{\nu_{y}} G(x, \cdot)\right|_{\partial M}$ determines the Green function $G(x, \cdot)$ in $M$ by elliptic unique continuation. Thus, instead of identifying points of $M$ with the corresponding Green functions in $M$ as in [28], we use the normal derivatives of the Green functions on $\partial M$. This change of point of view allows one to work on the boundary, which is natural since the measurements are given on $\partial M$.

The problem of finding the isometry, or conformal mapping if $n=2$, from the knowledge of the DN map is known as the geometric Calderón problem. This problem has been solved in [28] in the following cases:

Theorem Let $(M, g)$ be a compact connected $C^{\infty}$ Riemannian manifold with $C^{\infty}$ boundary.

(a) If $n=2$, the DN map $\Lambda_{g}$ determines the conformal class of $(M, g)$.

(b) If $n \geq 3$ and if $M, \partial M$ and $g$ are real-analytic, then the DN map $\Lambda_{g}$ determines $(M, g)$.

As a first application of our techniques we give new proofs of these results. The proofs consist of three steps.

(1) The first step is to determine the harmonic functions near the boundary, using a standard boundary determination result [30] and real-analyticity.

(2) The second step uses a unique continuation argument for harmonic functions where the manifold and harmonic functions are continued simultaneously. The use of harmonic coordinates (see e.g. [9]) and the Runge approximation property are key ingredients in this step. 
(3) Finally, we show that we can read the metric (conformal metric if $n=2$ ) from the knowledge of harmonic functions.

The works $[27,28,30]$ study the Calderón problem on real-analytic manifolds, and an analogous result for Einstein manifolds (which are real-analytic in the interior) is proved in [20]. See [26] for a recent result for a conformal Calderón problem. It remains a major open problem to remove the real-analyticity condition in dimensions $\geq 3$; for recent progress in the case of conformally transversally anisotropic manifolds see $[10,12]$ and also $[11,21]$ for the linearized problem. Other interesting approaches for related problems may be found in [2] and [5], and counterexamples for disjoint data are given in [7].

The inverse problems may not be uniquely solvable even when the metric is a priori known to be real analytic. Indeed, the above theorem proven in [28] does not hold for non-compact manifolds in the two-dimensional case. It is shown in [27] that there are a compact and a non-compact complete, two-dimensional manifold for which the boundary measurements are the same. This counterexample was obtained using a blow-up map. Analogous non-uniqueness results have been studied in the invisibility cloaking, where an arbitrary object is hidden from measurements by coating it with a material that corresponds to a degenerate Riemannian metric [8,15-18].

Our main tool for studying the Poisson embedding and constructing the metric from harmonic functions is the Runge approximation property. This property allows one to approximate local solutions to an elliptic equation by global solutions. In particular this implies that harmonic functions separate points and have prescribed Taylor expansions modulo natural constraints. There are many results of this type in the literature, see e.g. $[4,29,31,34]$. We require specific approximation results for uniformly elliptic operators, and for completeness they will be given in Appendix A together with proofs.

\subsection{An inverse problem for quasilinear equations}

As a new result we prove a determination result for Calderón type inverse problems for quasilinear equations on manifolds $(M, g)$ with boundary $\partial M$, including models that are both anisotropic and nonlinear. We consider the equation

$$
Q(u)=f \text { in } M, \quad u=0 \text { on } \partial M,
$$

where $f \in C_{c}^{\infty}(W), W \subset M$ is a fixed open set, and $Q$ is a uniformly elliptic quasilinear operator of the form

$$
Q u(x)=\mathcal{A}^{a b}(x, u(x), d u(x)) \nabla_{a} \nabla_{b} u(x)+\mathcal{B}(x, u(x), d u(x)) .
$$

Here and below we use Einstein summation rule, which means that repeated indices are always summed over $1, \ldots, n$. The source-to-solution mapping $S: C_{c}^{\infty}(W) \rightarrow$ $C^{\infty}(W)$ for this problem is defined as

$$
S(f)=\left.u_{f}\right|_{W}
$$


where $u_{f}$ solves (3). We assume that $(M, g)$ and the matrix valued function $\mathcal{A}$ and the function $\mathcal{B}$ are real analytic. In this case we show that the source-to-solution mapping, even for small data, determines the manifold and the coefficients $\mathcal{A}$ and $\mathcal{B}$ up to a diffeomorphism and a built in "gauge symmetry" of the problem:

Theorem 1.1 Let $\left(M_{1}, g_{1}\right)$ and $\left(M_{2}, g_{2}\right)$ be compact connected real analytic Riemannian manifolds with mutual boundary and assume that $Q_{j}, j=1,2$, are quasilinear uniformly elliptic operators of the form (4) satisfying (22)-(27). Assume that the coefficients $\mathcal{A}_{j}, \mathcal{B}_{j}$ are real analytic in all their arguments.

Let $W_{j} \subset M_{j}^{\mathrm{int}}, j=1,2$, be open sets so that there is a diffeomorphism $\phi: W_{1} \rightarrow$ $W_{2}$, and assume that the local source-to-solution maps $S_{j}$ for $Q_{j}$ on $W_{j}$ agree,

$$
\phi^{*} S_{2} f=S_{1} \phi^{*} f
$$

for small $f \in C_{c}^{\infty}\left(W_{2}\right)$.

Then there is a real analytic diffeomorphism $J: M_{1}^{\text {int }} \rightarrow M_{2}^{\text {int }}$ such that

$$
\mathcal{A}_{1}=J^{*} \mathcal{A}_{2}:=\mathcal{A}
$$

and

$$
\mathcal{B}_{1}-J^{*} \mathcal{B}_{2}=\mathcal{A}^{a b}\left(\Gamma(g)_{a b}^{k}-\Gamma\left(J^{*} g\right)_{a b}^{k}\right) \sigma_{k}
$$

The mapping $J$ satisfies

$$
\left.J\right|_{W_{1}}=\phi: W_{1} \rightarrow W_{2}
$$

In Theorem 1.1, the maps $\mathcal{A}, \mathcal{B}_{1}$ and $J^{*} \mathcal{B}_{2}$ have $(x, c, \sigma) \in M_{1} \times \mathbb{R} \times T_{x}^{*} M_{1}$ as their argument, and $\Gamma(g)_{a b}^{k}$ and $\Gamma\left(J^{*} g\right)_{a b}^{k}$ refers to the Christoffel symbols of the metrics $g$ and $J^{*} g$ respectively.

The reason why $\mathcal{B}$ can not be determined independently of $\mathcal{A}$ and $\Gamma$, as presented in (5), is due to the fact that the covariant Hessian in the definition of $Q$ contains first order terms.

The proof proceeds by linearizing the problem and using a slightly modified Poisson embedding for the linearized equation. Using the linearization we can first use the Poisson embedding approach to construct the manifold, but not yet the coefficients $\mathcal{A}$ and $\mathcal{B}$. The source-to-solution mapping determines the coefficients in the measurement set $W$. Since the manifold is now known, the proof is completed by determining the coefficients on the whole manifold by analytic continuation from the set $W$. The linearization method goes back to [24] and has been used in various inverse problems for nonlinear equations, including anisotropic problems [22,37]. We refer to [35,36] for further references. 


\subsection{Further aspects of Poisson embedding}

The governing principle of this paper is that instead of trying to find the metric in the anisotropic Calderón problem directly, one can focus on finding the harmonic functions. This principle is implemented by Poisson embedding and by the fact, which we prove, that the metric can be determined from the knowledge of harmonic functions. We will now discuss in more detail some aspects the Poisson embedding approach.

If $J$ is an isometry $\left(M_{1}, g_{1}\right) \rightarrow\left(M_{2}, g_{2}\right)$, then $J$ can be locally represented in various coordinate charts. Useful coordinate charts include boundary normal coordinates and harmonic coordinates [9,39]. In the study of the Calderón problem, boundary normal coordinates have been used to locally identify real analytic manifolds near boundary points by showing that in boundary normal coordinates the metrics $g_{1}$ and $g_{2}$ agree. See e.g. [20,26-28,30]. However, local representations do not yield a global candidate for the isometry $J$ required for solving the Calderón problem. In contrast, the Poisson embeddings $P_{j}$ are globally defined objects, and they yield a candidate $P_{2}^{-1} \circ P_{1}$ for the isometry (in fact we prove that if $P_{2}^{-1} \circ P_{1}$ is well defined, then it gives the required isometry). The representation formula $P_{2}^{-1} \circ P_{1}$ also gives uniqueness of the boundary fixing isometry directly if it exists.

In [27] the authors introduce an embedding of real analytic Riemannian manifolds by using Green functions to study the Calderón problem. Their approach involves an analytic continuation argument based on the implicit function theorem applied to the embedding. In contrast, the analogous step for Poisson embedding can be done simply by using harmonic coordinates. This feature also emphasizes the role of choosing suitable coordinates in the study of the anisotropic Calderón problem. Moreover, the recovery of the metric using Poisson embedding is an elementary linear algebra argument that yields a representation of the metric in terms of harmonic functions (see Proposition 4.1). In [27] an asymptotic expansion of Green functions near the diagonal is used.

The basic principle of Poisson embedding is to control the points on a manifold by the values of solutions to Dirichlet problems on the manifold. This principle generalizes to nonlinear equations, to linear systems or to nonlocal operators where Green functions might not be easily accessible. The Poisson embedding also generalizes directly to less regular, say piecewise smooth or $C^{k}$ regular, settings.

Finally, we mention that ideas related to the Poisson embedding have been used in other fields as well. In [19] an embedding by finitely many harmonic functions is used to embed an open Riemannian manifold into a higher dimensional Euclidean space. Their embedding is similar to the Poisson embedding, in the sense that the Poisson embedding parametrizes the manifold by the data of all, instead of a finite number, of harmonic functions on the manifold. Another related result is that when the mapping $P_{2}^{-1} \circ P_{1}: M_{1} \rightarrow M_{2}$ exists, it is necessarily a harmonic morphism. The study of harmonic morphisms, which are mappings that preserve harmonic functions, has applications in the study of minimal surfaces and in mathematical physics [1]. In Sect. 4 we give a new proof of the characterization of harmonic morphisms as homotheties [1, Corollary 3.5.2] based on harmonic coordinates. 


\subsection{Outline of the paper}

In Sect. 2 we introduce the Poisson embedding and its basic properties, in a way that does not directly involve the Calderón problem. In Sect. 3 we determine the harmonic functions from the knowledge of the DN map on real analytic manifolds by using the Poisson embedding. From the knowledge of harmonic functions, we then determine the metric in Sect. 4, which gives a new proof of the main result of [30] in dimension $n \geq 3$. Section 5 gives a new proof of the two-dimensional result of [30]. In Sect. 6 we use the Poisson embedding approach and linearization to prove Theorem 1.1, yielding uniqueness in the inverse problem for quasilinear equations. Appendix A is independent of the rest of the paper and contains Runge approximation results.

\section{Poisson embedding}

We begin by introducing the Poisson embedding of a Riemannian manifold $(M, g)$ with boundary. Throughout this section, we assume that $M$ is connected and $M$ and $g$ are $C^{\infty}$. In particular, we do not require real analyticity in the definition of the Poisson embedding. We will solve Dirichlet problems with boundary values supported on a nonempty open set $\Gamma$ of the boundary $\partial M$. The domain of the Poisson embedding will be

$$
M^{\Gamma}:=M^{\text {int }} \cup \Gamma \subset M
$$

Definition 2.1 (Poisson embedding) Let $(M, g)$ be a compact Riemannian manifold with boundary, and let $\Gamma$ be a nonempty open subset of $\partial M$. The Poisson embedding of the manifold $M$ is defined to be the mapping

$$
P: M^{\Gamma} \rightarrow \mathcal{D}^{\prime}(\Gamma), \quad P(x) f=u_{f}(x)
$$

where $u_{f}(x)$ solves the Dirichlet problem

$$
\begin{aligned}
\Delta_{g} u_{f} & =0 \text { in } M, \\
u_{f} & =f \text { on } \partial M
\end{aligned}
$$

with $f \in C_{c}^{\infty}(\Gamma)$.

In the definition $\mathcal{D}^{\prime}(\Gamma)$ is the space of distributions on $\Gamma$, i.e. $\mathcal{D}^{\prime}(\Gamma)$ is the dual of $C_{c}^{\infty}(\Gamma)$. We call $P$ the Poisson embedding due to the connection with the representation formula for the solution $u_{f}(x)$ in terms of the Poisson kernel

$$
\partial_{\nu_{y}} G(x, y)
$$

where $x \in M$ and $y \in \partial M$, as

$$
u_{f}(x)=\int_{\partial M} \partial_{\nu_{y}} G(x, y) f(y) d S_{g_{\partial M}} .
$$


Here $G(x, y)$ is the Dirichlet Green's function of $(M, g)$ and $d S_{g_{\partial M}}$ is the induced metric on the boundary. Thus $P(x)$ can be identified with the distribution $\partial_{v_{y}} G(x, \cdot)$ on $\Gamma$. In fact, if $x \in M^{\text {int }}$ then $\partial_{\nu_{y}} G(x, \cdot)$ is in $C^{\infty}(\Gamma)$, and if $x \in \Gamma$ then $\partial_{\nu_{y}} G(x, \cdot)=$ $\delta_{x}(\cdot)$ so $P(x)$ is always a measure on $\Gamma$.

We have the following basic properties of $P$. Below, for $s \in \mathbb{R}$, we write $H^{s}(\partial M)$ for the standard $L^{2}$ based Sobolev space on $\partial M$, and $H^{s}(\Gamma)$ is defined by restriction, i.e. $H^{s}(\Gamma)=\left\{\left.f\right|_{\Gamma} ; f \in H^{s}(\partial M)\right\}$.

Proposition 2.1 Let $(M, g)$ be a compact manifold with boundary. For any $x \in M^{\Gamma}$, one has $P(x) \in H^{-s}(\Gamma)$ whenever $s+1 / 2>n / 2$. The mapping $P$ is continuous $M^{\Gamma} \rightarrow H^{-s-1}(\Gamma)$ and $k$ times Fréchet differentiable considered as a mapping $M^{\Gamma} \rightarrow$ $H^{-s-1-k}(\Gamma)$. In particular, $P: M^{\Gamma} \rightarrow \mathcal{D}^{\prime}(\Gamma)$ is $C^{\infty}$ smooth in the Fréchet sense.

The Fréchet derivative of $P$ at $x$ is a linear mapping given by

$$
D P_{x}: T_{x} M^{\Gamma} \rightarrow \mathcal{D}^{\prime}(\Gamma), \quad\left(D P_{x} V\right) f=d u_{f}(x) \cdot V
$$

where $u_{f}$ solves $\Delta_{g} u_{f}=0$ in $M$ and $\left.u_{f}\right|_{\partial M}=f \in C_{c}^{\infty}(\Gamma)$.

In the proposition - refers to the canonical pairing of vectors and covectors on $M^{\Gamma}$, that is, $d u_{f}(x) \cdot V \equiv\left[d u_{f}(x)\right](V)=\partial_{a} u_{f}(x) V^{a}$.

We omit the proof of Proposition 2.1, which is just an application of standard estimates for solutions of elliptic equations. However, let us formally calculate where the formula for the derivative of $P$ comes from. Let $x \in M^{\Gamma}$ and $V \in T_{x} M^{\Gamma}$. By definition $V$ is given by a path $\gamma:[0,1] \rightarrow M^{\Gamma}$ such that $\gamma(0)=x$ and $\left.\frac{d}{d t}\right|_{t=0} \gamma(t)=V$. Let $f \in C_{c}^{\infty}(\Gamma)$. A formal calculation of $D P_{x} V \in \mathcal{D}^{\prime}(\Gamma)$ now gives

$\left[D P_{x} V\right] f=\left[\left.\frac{d}{d t}\right|_{t=0} P \circ \gamma(t)\right] f=\left.\frac{d}{d t}\right|_{t=0} u_{f}(\gamma(t))=\partial_{a} u_{f}(x) V^{a}=d u_{f}(x) \cdot V$.

Let us next show that the mapping $P$ is indeed an embedding, which means a $C^{\infty}$ injective mapping with injective Fréchet derivative. The main tool that we encounter here for the first time is Runge approximation, which allows one to approximate locally defined harmonic functions by global harmonic functions. It is known since [29,31] that approximation results of this type follow by duality from the unique continuation principle. We have devoted Appendix A to various Runge approximation results. We will mostly use the following consequence, whose proof may also be found in Appendix A.

Proposition 2.2 Let $(M, g)$ be a compact Riemannian manifold with smooth boundary, and let $\Gamma$ be a nonempty open subset of $\partial M$.

(a) If $x \in M^{\Gamma}, y \in M$ and $x \neq y$, there is $f \in C_{c}^{\infty}(\Gamma)$ such that

$$
u_{f}(x) \neq u_{f}(y) .
$$


(b) If $x \in M^{\Gamma}$ and $v \in T_{x}^{*} M$, there is $f \in C_{c}^{\infty}(\Gamma)$ such that

$$
d u_{f}(x)=v .
$$

Proposition 2.3 ( $P$ is an embedding) Let $(M, g)$ be a compact manifold with boundary. The mapping $P: M^{\Gamma} \rightarrow \mathcal{D}^{\prime}(\Gamma)$ is a $C^{\infty}$ embedding in the sense that it is injective with injective Fréchet derivative on $T M^{\Gamma}$.

Proof Let $x_{1}, x_{2} \in M^{\Gamma}$ and assume that $P\left(x_{1}\right)=P\left(x_{2}\right)$. That is, for all boundary value functions $f \in C_{c}^{\infty}(\Gamma)$, we have

$$
u_{f}\left(x_{1}\right)=P_{1}\left(x_{1}\right) f=P_{2}\left(x_{2}\right) f=u_{f}\left(x_{2}\right) .
$$

We need to show that $x_{1}=x_{2}$. We argue by contradiction and suppose that $x_{1} \neq x_{2}$. But by Proposition 2.2 there is $f_{0} \in C_{c}^{\infty}(\Gamma)$ such that

$$
u_{f_{0}}\left(x_{1}\right) \neq u_{f_{0}}\left(x_{2}\right) \text {. }
$$

This is a contradiction and we must have $x_{1}=x_{2}$. Thus $P$ is injective.

To show that the differential of $P$ is injective, let $x \in M^{\Gamma}$ and $V \in T_{x} M^{\Gamma}$, and assume that $D P_{x} V=0$. By the formula (6) for the differential $D P_{x}$, we have

$$
\left[D P_{x} V\right] f=d u_{f}(x) \cdot V=0,
$$

for all boundary value functions $f \in C_{c}^{\infty}(\Gamma)$. To conclude that $V=0$, we use Proposition 2.2 again and choose $f \in C_{c}^{\infty}(\Gamma)$ such that $\nabla u_{f}(x)=V$. Here $\nabla u$ is the Riemannian gradient. Thus the condition (7) yields

$$
0=d u_{f}(x) \cdot V=|V|_{g(x)}^{2}
$$

showing that $V=0$. Thus the differential of $P$ is injective on $T M^{\Gamma}$.

\subsection{Composition of Poisson embeddings}

Let $\left(M_{1}, g_{1}\right)$ and $\left(M_{2}, g_{2}\right)$ be compact manifolds with mutual boundary $\partial M$. One of the aims of this paper is to give a candidate for the isometry that one hopes to construct in the anisotropic Calderón problem. This candidate is

$$
J:=P_{2}^{-1} \circ P_{1}
$$

We will see that, whenever this mapping is well defined from $M_{1}$ to $M_{2}$, it is exactly the mapping that one seeks in the Calderón problem. In dimensions $n \geq 3$, it is an isometry. In dimension 2 , it is a conformal mapping. It also fixes the boundary if we consider full data problem, or the part $\Gamma \subset \partial M$, if we consider partial data problem with measurements made on $\Gamma$. 
We now begin to study the composition $P_{2}^{-1} \circ P_{1}$. We include considerations related to partial data problem on the part $\Gamma$ of the boundary. For this purpose we denote throughout this section

$$
M_{1}^{\Gamma}=M_{1}^{\text {int }} \cup \Gamma \text { and } M_{2}^{\Gamma}=M_{2}^{\text {int }} \cup \Gamma .
$$

Since we already know that the Poisson embeddings $P_{j}, j=1,2$, are injective, we know that the mapping is well defined and bijective if the image sets of $P_{j}$ : $\left(M_{j}^{\Gamma}, g_{j}\right) \rightarrow \mathcal{D}^{\prime}(\Gamma)$ agree:

$$
P_{1}\left(M_{1}^{\Gamma}\right)=P_{2}\left(M_{2}^{\Gamma}\right)
$$

Thus solving the Calderón problem would reduce to verifying this condition from the knowledge that the DN maps of $\left(M_{1}, g_{1}\right)$ and $\left(M_{2}, g_{2}\right)$ agree.

The next lemma considers the smoothness properties of the mapping $J$ assuming it is defined on some open set of $M_{1}$.

Lemma 2.4 Let $\left(M_{j}, g_{j}\right), j=1,2$, be compact manifolds with mutual boundary $\partial M$. Assume that

$$
P_{1}(B) \subset P_{2}\left(M_{2}^{\Gamma}\right)
$$

for some open set $B \subset M_{1}^{\Gamma}$. Then $J(B) \subset M_{2}^{\Gamma}$, and $J=P_{2}^{-1} \circ P_{1}$ is a $C^{\infty}$ diffeomorphism $B \rightarrow J(B)$.

Proof We first note that, writing $u_{f}^{j}(x)=P_{j}(x) f$, for any $f \in C_{c}^{\infty}(\Gamma)$ one has

$$
u_{f}^{1}(x)=u_{f}^{2}(J(x)), \quad x \in B
$$

This follows from the computation $P_{1}(x)=P_{2}\left(P_{2}^{-1} \circ P_{1}(x)\right)=P_{2} \circ J(x)$. Now, to show that $J(B) \subset M_{2}^{\Gamma}$, we argue by contradiction and assume that there is some $x \in B \subset M_{1}^{\Gamma}$ with $J(x) \in \partial M \backslash \Gamma$. But then by (8)

$$
u_{f}^{1}(x)=u_{f}^{2}(J(x))=0, \quad f \in C_{c}^{\infty}(\Gamma),
$$

which is impossible by Proposition 2.2. Thus $J(B) \subset M_{2}^{\Gamma}$.

We next prove that $J: B \rightarrow M_{2}$ is continuous (this perhaps surprisingly uses compactness of $M_{2}$ ). Let $x \in B$. If $J$ would not be continuous at $x$, there would be $\varepsilon>0$ and a sequence $\left(x_{l}\right) \subset M_{1}$ with $x_{l} \rightarrow x$ such that $J\left(x_{l}\right) \notin B(J(x), \varepsilon)$. By the compactness of $M_{2}$, passing to a subsequence (still denoted by $\left(x_{l}\right)$ ), we may assume that $J\left(x_{l}\right)$ converges to $y \in M_{2}$. We have $d(y, J(x)) \geq \varepsilon$.

Now, using Proposition 2.2 in $M_{2}$ and the fact that $J(x) \in M_{2}^{\Gamma}$, we can find $f \in$ $C_{c}^{\infty}(\Gamma)$ such that the harmonic function $u_{f}^{2} \in C^{\infty}\left(M_{2}\right)$ satisfies $u_{f}^{2}(J(x)) \neq u_{f}^{2}(y)$. The formula (8) shows that

$$
u_{f}^{1}\left(x_{l}\right)-u_{f}^{1}(x)=u_{f}^{2}\left(J\left(x_{l}\right)\right)-u_{f}^{2}(J(x)) .
$$


Since harmonic functions are continuous, taking the limit $l \rightarrow \infty$ yields

$$
0=u_{f}^{2}(y)-u_{f}^{2}(J(x)),
$$

which is a contradiction. Thus $J$ is continuous.

We will next show that $J: B \rightarrow M_{2}$ is $C^{\infty}$. This follows an idea from [39] related to smoothness of Riemannian isometries. Fix $x \in B$, and choose harmonic coordinates $U=\left(u_{f_{1}}^{2}, \ldots, u_{f_{n}}^{2}\right)$ with $f_{j} \in C_{c}^{\infty}(\Gamma), j=1, \ldots, n$, near $J(x)$. This can be done by Proposition 2.2 upon choosing $\left\{d u_{f_{1}}^{2}(J(x)), \ldots, d u_{f_{n}}^{2}(J(x))\right\}$ linearly independent. Write $V=\left(u_{f_{1}}^{1}, \ldots, u_{f_{n}}^{2}\right)$. By the formula (8) we have $V=U \circ J$ in $B$. Now $U$ is bijective in some neighborhood $\Omega \subset M_{2}$ of $J(x)$, and since $J$ is continuous there is a neighborhood $W$ of $x$ with $J(W) \subset \Omega$. Thus we have

$$
J=U^{-1} \circ V \text { in } W .
$$

Since the harmonic functions $u_{f_{k}}^{j}, j=1,2$, are smooth, the smoothness of $J$ near $x$ follows.

It remains to show that the differential of $J$ is invertible on $B$. Since $J: B \rightarrow M_{2}$ is injective, the claim will then follow from the inverse function theorem. Let $f \in$ $C_{c}^{\infty}(\Gamma), x \in B$ and $X \in T_{x} M_{1}$. By (8), we have $u_{f}^{1}=u_{f}^{2} \circ J$. Together with (6) this gives:

$$
\begin{aligned}
{\left[D P_{1}(x) X\right] f } & =X \cdot d u_{f}^{1}(x)=X \cdot d\left(u_{f}^{2} \circ J\right)(x)=X \cdot\left(\left.d u_{f}^{2}\right|_{J(x)}(D J)^{T}\right) \\
& =\left.d u_{f}^{2}\right|_{J(x)} \cdot(D J(x)) X .
\end{aligned}
$$

The left hand side is equal to $d u_{f}^{1} \cdot X$. Thus for any $f \in C_{c}^{\infty}(\Gamma)$ we have the equation

$$
\left.d u_{f}^{1}\right|_{x} \cdot X=\left.d u_{f}^{2}\right|_{J(x)} \cdot(D J(x)) X .
$$

This equation will be used again later on and we name it "the equation of injectivity". (This equation can be interpreted as the infinite dimensional counterpart of the chain rule for the composition $P_{2} \circ\left(P_{2}^{-1} \circ P_{1}\right)$.)

Assume that $(D J(x)) X=0$ and choose by Proposition 2.2 a harmonic function $u_{f}^{1}$ so that the Riemannian gradient satisfies $\nabla u_{f}^{1}(x)=X$. Then

$$
|X|^{2}=d u_{f}^{2} \cdot(D J(x)) X=0 .
$$

Thus $X=0$. It follows that $D J(x)$ is injective at $x$ and since the manifolds are of the same dimension, it is invertible. This proves the claim.

\section{Determination of harmonic functions}

We have so far acquired the basic properties of the Poisson embedding. We now move on to give a new proof of the fact [28] that for real analytic Riemannian manifolds 
with $\operatorname{dim}(M) \geq 3$, the knowledge of the DN-map determines the Riemannian manifold up to isometry. Throughout this section we will assume that the manifolds $M_{j}$ and metrics $g_{j}, j=1,2$, are real analytic, and $n=\operatorname{dim}\left(M_{j}\right) \geq 3$. We continue to denote $M_{j}^{\Gamma}=M_{j}^{\text {int }} \cup \Gamma$.

We first show that near any boundary point there exist coordinates in which the coordinate representations of harmonic functions, corresponding to a common boundary value $f$, agree. This follows from boundary determination [30] and unique continuation.

Lemma 3.1 (Determination near the boundary) Let $\left(M_{1}, g_{1}\right)$ and $\left(M_{2}, g_{2}\right)$ be compact real analytic manifolds with mutual boundary whose DN maps agree on an open set $\Gamma \subset \partial M$. Assume also that $\Gamma$ is real analytic. Then, for any $p \in \Gamma$ there are boundary normal coordinates $\psi_{j}, j=1,2$, defined on neighborhoods $U_{j} \subset M_{j}^{\Gamma}$ of $p$, such that $\psi_{1}$ and $\psi_{2}$ agree on $\Gamma$ and such that for any boundary function $f \in C_{c}^{\infty}(\Gamma)$ we have

$$
u_{f}^{1} \circ \psi_{1}^{-1}(x)=u_{f}^{2} \circ \psi_{2}^{-1}(x), \quad x \in \psi_{1}\left(U_{1}\right) \cap \psi_{2}\left(U_{2}\right) \subset\left\{x^{n} \geq 0\right\} .
$$

Here the functions $u_{f}^{j} \circ \psi_{j}^{-1}$ are the coordinate representations of the harmonic functions $u_{f}^{j}$ on $\left(M_{j}, g_{j}\right)$ with boundary value $f$.

Proof Let $p \in \Gamma \subset \partial M$ and let $\psi_{j}, j=1,2$, be boundary normal coordinates near $p$ on manifolds $\left(M_{j}, g_{j}\right)$, respectively, so that $\left.\psi_{1}\right|_{\Gamma}=\left.\psi_{2}\right|_{\Gamma}$. Then by the boundary determination result in [30], we have that in these coordinates, the jets of the Riemannian metrics $g_{j}$ agree. Since $g_{j}$ and $\Gamma$ are real analytic, it follows that $\psi_{j}$ are real analytic coordinate charts, and thus the coordinate representations $\psi_{j}^{-1 *} g_{j}$ of $g_{j}$ agree near $x=\psi_{1}(p)=\psi_{2}(p) \in\left\{x_{n}=0\right\}$.

Write $g=\psi_{1}^{-1 *} g_{1}=\psi_{2}^{-1 *} g_{2}$. If $f$ is any $C_{c}^{\infty}(\Gamma)$ boundary function, we have that $\tilde{u}_{f}^{1}=u_{f}^{1} \circ \psi_{1}^{-1}(x)$ and $\tilde{u}_{f}^{2}=u_{f}^{2} \circ \psi_{2}^{-1}(x)$ satisfy the same elliptic equation

$$
\Delta_{g} \tilde{u}_{f}^{i}=0 \text { in } \psi_{1}\left(U_{1}\right) \cap \psi_{2}\left(U_{2}\right)
$$

with the same Cauchy data (since the DN-maps agree)

$$
\tilde{u}_{f}^{1}=\tilde{u}_{f}^{2}, \quad \partial_{x_{n}} \tilde{u}_{f}^{1}=\partial_{x_{n}} \tilde{u}_{f}^{2} \text { on } \psi_{1}(\Gamma) \cap \psi_{2}(\Gamma) .
$$

Thus by elliptic unique continuation [25, Theorem 3.3.1], we have

$$
\tilde{u}_{f}^{1}=\tilde{u}_{f}^{2} \text { on } \psi_{1}\left(U_{1}\right) \cap \psi_{2}\left(U_{2}\right)
$$

as claimed.

Lemma 3.2 Under the same assumptions as in Lemma 3.1, there exists an open set $B \subset M_{1}^{\Gamma}$, which contains all points of $\Gamma$, and a $C^{\infty}$ diffeomorphism $F: B \rightarrow F(B) \subset$ $M_{2}^{\Gamma}$ such that

$$
P_{1}(B) \subset P_{2}\left(M_{2}^{\Gamma}\right), \quad P_{1}=P_{2} \circ F \text { on } B .
$$


Proof By Lemma 3.1, in its notation, we have that for any $p \in \Gamma$ there exist $U_{j} \subset M_{j}^{\Gamma}$, $j=1,2$, such that for all $f \in C_{c}^{\infty}(\Gamma)$, we have

$$
u_{f}^{1} \circ \psi_{1}^{-1}=u_{f}^{2} \circ \psi_{2}^{-1} \text { on } \psi_{1}\left(U_{1}\right) \cap \psi_{2}\left(U_{2}\right) .
$$

Thus for $x \in \psi_{1}^{-1}\left(\psi_{1}\left(U_{1}\right) \cap \psi_{2}\left(U_{2}\right)\right)$, we have

$$
P_{1}(x) f=u_{f}^{1}(x)=u_{f}^{2}\left(\psi_{2}^{-1} \circ \psi_{1}(x)\right)=P_{2}\left(\psi_{2}^{-1} \circ \psi_{1}(x)\right) f .
$$

Setting $B_{p}=\psi_{1}^{-1}\left(\psi_{1}\left(U_{1}\right) \cap \psi_{2}\left(U_{2}\right)\right)$ and

$$
B=\bigcup_{p \in \Gamma} B_{p}
$$

gives an open set $B \subset M_{1}^{\Gamma}$ such that $\Gamma \subset B$ and $P_{1}(B) \subset P_{2}\left(M_{2}^{\Gamma}\right)$. By Lemma 2.4 it is enough to set $F=P_{2}^{-1} \circ P_{1}$ in $B$.

We have now shown that knowledge of the DN map on $\Gamma$ determines harmonic functions near $\Gamma$. We proceed in the real analytic case to determine the harmonic functions globally. From this knowledge we will then determine Riemannian manifolds up to isometry in Sect. 4.

In the following result a (global) harmonic morphism means a mapping that preserves solutions to the Dirichlet problem. Precisely, a $C^{\infty}$ mapping $H:\left(M_{1}^{\Gamma}, g_{1}\right) \rightarrow$ $\left(M_{2}^{\Gamma}, g_{2}\right)$ is a harmonic morphism, if for any $f \in C_{c}^{\infty}(\Gamma)$ we have

$$
u_{f}^{1}=u_{f}^{2} \circ H .
$$

Here $u_{f}^{1}$ and $u_{f}^{2}$ are the solutions to the Dirichlet problems for $\Delta_{g_{1}}$ and $\Delta_{g_{2}}$ as usual. For more details on harmonic morphisms, we refer to [1]. (In [1] a harmonic morphism is a mapping that also preserves local harmonic functions instead of just global harmonic functions, but our results will show that there is no difference at least when $\operatorname{dim}\left(M_{1}\right)=$ $\operatorname{dim}\left(M_{2}\right)$.)

Theorem 3.3 Let $\left(M_{1}, g_{1}\right)$ and $\left(M_{2}, g_{2}\right)$ be compact real analytic manifolds with mutual boundary. Let $\Gamma$ be an open subset of $\partial M$. Assume that there is a neighborhood $B \subset M_{1}^{\Gamma}$ of a boundary point $p \in \Gamma$ and a mapping $F: B \rightarrow F(B) \subset M_{2}^{\Gamma}$ diffeomorphic onto its image such that

$$
P_{1}=P_{2} \circ F \text { on } B \text {. }
$$

Then we have $P_{1}\left(M_{1}^{\Gamma}\right)=P_{2}\left(M_{2}^{\Gamma}\right)$ and

$$
J:=P_{2}^{-1} \circ P_{1}: M_{1}^{\Gamma} \rightarrow M_{2}^{\Gamma}
$$

is a diffeomorphic global harmonic morphism extending $F$. 
Proof Redefine $B$ to be the largest connected open subset of $M_{1}^{\Gamma}$ such that $P_{1}(B) \subset$ $P_{2}\left(M_{2}^{\Gamma}\right)$. By our assumption $B$ is nonempty. We will show that $B$ is closed and thus $B=M_{1}^{\Gamma}$. Then $P_{1}\left(M_{1}^{\Gamma}\right) \subset P_{2}\left(M_{2}^{\Gamma}\right)$, and from Lemma 2.4 it will follow that

$$
J=P_{2}^{-1} \circ P_{1}: M_{1}^{\Gamma} \rightarrow M_{2}^{\Gamma}
$$

is a well defined $C^{\infty}$ diffeomorphism. It is also a harmonic morphism, since for any $f \in C_{c}^{\infty}(\Gamma)$ and $x \in M_{1}^{\Gamma}$ we would have

$$
J(x)=P_{2}^{-1}\left(P_{1}(x)\right) \Rightarrow P_{2}(J(x)) f=P_{1}(x) f \Longleftrightarrow u_{f}^{2}(J(x))=u_{f}^{1}(x) .
$$

This would prove the claim.

We argue by contradiction and assume that $B$ is not closed. Then there is a sequence $\left(p_{k}\right)$ in $B$ with $p_{k} \rightarrow x_{1}$ as $k \rightarrow \infty$, where $x_{1} \in \partial B \backslash B \subset M_{1}^{\Gamma}$. After passing to a subsequence, there is $x_{2} \in M_{2}$ such that

$$
x_{2}=\lim _{k \rightarrow \infty} J\left(p_{k}\right)
$$

We actually have that $x_{2} \in M_{2}^{\Gamma}$. This is because if $x_{2} \in \partial M \backslash \Gamma$, then (8) applied at $x=p_{k}$ shows that for all $f \in C_{c}^{\infty}(\Gamma)$ we have

$$
u_{f}^{1}\left(x_{1}\right)=\lim u_{f}^{1}\left(p_{k}\right)=\lim u_{f}^{2}\left(J\left(p_{k}\right)\right)=u_{f}^{2}\left(x_{2}\right)=f\left(x_{2}\right)=0 .
$$

This cannot be true by Proposition 2.2, so indeed $x_{2}$ must be in $M_{2}^{\Gamma}$.

Let

$$
U=\left(u_{f_{1}}^{2}, u_{f_{2}}^{2}, \ldots, u_{f_{n}}^{2}\right)
$$

be harmonic coordinates on a neighborhood $\Omega_{2} \subset M_{2}^{\Gamma}$ of $x_{2}$, as in Lemma 2.4. Here $u_{f_{l}}^{2}, l=1, \ldots, n$, are global harmonic functions on $M_{2}$ with boundary values $f_{l} \in C_{c}^{\infty}(\Gamma)$.

The mapping $J=P_{2}^{-1} \circ P_{1}: B \rightarrow J(B)$ is well defined by Lemma 2.4. Now comes the punch line: we set

$$
V=\left(u_{f_{1}}^{1}, u_{f_{2}}^{1}, \ldots, u_{f_{n}}^{1}\right)
$$

We will prove shortly that $V$ is a coordinate system in some neighborhood $\Omega_{1} \subset M_{1}^{\Gamma}$ of $x_{1}$. The map $U^{-1} \circ V$ will then give us a local identification of neighborhoods $\Omega_{1} \subset M_{1}^{\Gamma}$ of $x_{1}$ and $\Omega_{2} \subset M_{2}^{\Gamma}$ of $x_{2}$, such that $\Omega_{1}$ intersects the complement of the closure of $B$ in $M_{1}^{\Gamma}$ (if nonempty). This will allow us to extend $B$ and reach a contradiction.

We now take a small deviation from the main line of the proof, and show that $V$ is also a harmonic coordinate system around $x_{1}$. To see this, first observe that 
$V\left(x_{1}\right)=U\left(x_{2}\right)$ since

$$
u_{f_{l}}^{1}\left(x_{1}\right)=\lim _{k} u_{f_{l}}^{1}\left(p_{k}\right)=\lim _{k} u_{f_{l}}^{2}\left(J\left(p_{k}\right)\right)=u_{f_{l}}^{2}\left(x_{2}\right), \quad l=1, \ldots, n .
$$

Next we note that we have at $p_{k}$ the equation of injectivity (10)

$$
d u_{f}^{1}\left(p_{k}\right) \cdot X_{k}=\left.d u_{f}^{2}\right|_{J\left(p_{k}\right)} \cdot D\left(P_{2}^{-1} \circ P_{1}\right)\left(p_{k}\right) X_{k} .
$$

This holds for all $X_{k} \in T_{p_{k}} M_{1}$. Since near $x_{1}$ on $B$, we have $J=U^{-1} \circ V$, see (9), we can substitute this to the equation of injectivity yielding

$$
d u_{f}^{1}\left(p_{k}\right) \cdot X_{k}=\left.d u_{f}^{2}\right|_{J\left(p_{k}\right)} \cdot D\left(U^{-1} \circ V\right)\left(p_{k}\right) X_{k} .
$$

Assume that $X \in T_{x_{1}} M$ is such that $D\left(U^{-1} \circ V\right)\left(x_{1}\right) X=0$. Let us take a sequence $X_{k} \rightarrow X$ in $T M$ as $k \rightarrow \infty$. Note that $D\left(U^{-1} \circ V\right)$ is a continuous (in fact smooth) matrix field even though we still do not know if it is invertible. Taking the limit as $k \rightarrow \infty$ in (13) gives

$$
d u_{f}^{1}\left(x_{1}\right) \cdot X=d u_{f}^{2}\left(x_{2}\right) \cdot D\left(U^{-1} \circ V\right)\left(x_{1}\right) X .
$$

Choosing $f$ so that $\nabla u_{f}^{1}\left(x_{1}\right)=X$ (by Proposition 2.2) shows that $X=0$ and consequently that $D\left(U^{-1} \circ V\right)\left(x_{1}\right)$ is invertible. Since $U$ is a local diffeomorphism, it follows that $D V\left(x_{1}\right)$ is invertible. Thus $V$ is also a coordinate system near $x_{1}$ as claimed.

Let us continue on the main line of the proof of the proposition. Let $\Omega_{1} \subset M_{1}^{\Gamma}$ be a neighborhood of $x_{1}$ so that $V$ is a coordinate system in $\Omega_{1}$, and redefine $\Omega_{1}$, if necessary, so that $V\left(\Omega_{1}\right) \subset U\left(\Omega_{2}\right)$. On $B$ we have by (8)

$$
V=U \circ J
$$

What we will show is that

$$
P_{1}(x)=P_{2}\left(U^{-1} \circ V(x)\right), \quad x \in \Omega_{1} .
$$

Note that we require (15) to hold in $\Omega_{1}$, not only in $B \cap \Omega_{1}$. In particular we will have

$$
P_{1}\left(B \cup \Omega_{1}\right) \subset P_{2}\left(M_{2}^{\Gamma}\right) .
$$

Since $\Omega_{1}$ in this case extends $B$ to a neighborhood of the point $x_{1} \in \partial B \backslash B$, this will give a contradiction and prove the theorem since $M_{1}^{\Gamma}$ is connected.

So far we have not used the assumption of real analyticity, but we use it now to prove (15). To prove it, let $f \in C_{c}^{\infty}(\Gamma)$. Now $u_{f}^{j} \in C^{\omega}\left(M_{j}^{\text {int }}\right)$ and $V$ and $U$ are local $C^{\omega}$ diffeomorphisms in $\Omega_{1} \cap M_{1}^{\text {int }}$ and $\Omega_{2} \cap M_{2}^{\text {int }}$, respectively. Thus we have

$$
u_{f}^{1} \circ V^{-1}, u_{f}^{2} \circ U^{-1} \in C^{\omega}\left(V\left(\Omega_{1} \cap M_{1}^{\mathrm{int}}\right)\right)
$$


where $V\left(\Omega_{1} \cap M_{1}^{\text {int }}\right) \subset \mathbb{R}^{n}$. Since on $B \cap \Omega_{1}$, we have by (11) and (14) that

$$
u_{f}^{1}=u_{f}^{2} \circ J \text { and } J=U^{-1} \circ V
$$

it follows that

$$
u_{f}^{1} \circ V^{-1}=u_{f}^{2} \circ U^{-1} \text { on } V\left(B \cap \Omega_{1}\right)
$$

By the real analyticity, stated in (16), this holds on the whole $V\left(\Omega_{1}\right)$. This means that the coordinate representations of $u_{f}^{1}$ and $u_{f}^{2}$ in the (harmonic) coordinates $V$ and $U$ agree.

Given any $f \in C_{c}^{\infty}(\Gamma)$, we have the chain of equivalences

$$
\begin{aligned}
& P_{1}(x) f=P_{2}\left(U^{-1} \circ V(x)\right) f, x \in \Omega_{1} \\
\Longleftrightarrow & u_{f}^{1}(x)=u_{f}^{2}\left(U^{-1} \circ V(x)\right), x \in \Omega_{1} \\
\Longleftrightarrow & u_{f}^{1} \circ V^{-1}=u_{f}^{2} \circ U^{-1} \text { on } V\left(\Omega_{1}\right) \subset \mathbb{R}^{n} .
\end{aligned}
$$

Since we have proven the latter, and since the boundary function $f$ was arbitrary, we have proven (15). Consequently we have

$$
P_{1}\left(\Omega_{1}\right)=P_{2}\left(U^{-1}\left(V\left(\Omega_{1}\right)\right)\right) \subset P_{2}\left(\Omega_{2}\right) \subset P_{2}\left(M_{2}^{\Gamma}\right)
$$

Thus $B$ extends to a neighborhood of the point $x_{1} \in \partial B \backslash B$, which gives a contradiction. We have now proved that $B$ is closed. Since $M_{1}^{\Gamma}$ is connected, we conclude that $P_{1}\left(M_{1}^{\Gamma}\right) \subset P_{2}\left(M_{2}^{\Gamma}\right)$. We thus also have $J\left(M_{1}^{\Gamma}\right) \subset M_{2}^{\Gamma}$.

Inverting the role of $\left(M_{1}, g_{1}\right)$ and $\left(M_{2}, g_{2}\right)$, and replacing $F$ and $J$ in the statement of the theorem by $F^{-1}$ and $J^{-1}$, shows that $P_{1}\left(M_{1}^{\Gamma}\right)=P_{2}\left(M_{2}^{\Gamma}\right)$ and that $J$ is surjective onto $M_{2}^{\Gamma}$. Consequently $J: M_{1}^{\Gamma} \rightarrow M_{2}^{\Gamma}$ is diffeomorphism by Lemma 2.4. Since we already showed that $J$ is a global harmonic morphism at the beginning of the proof in (11), the claim follows.

Combining Lemma 3.2 and Theorem 3.3, we have proved the following statement.

Theorem 3.4 Let $\left(M_{1}, g_{1}\right)$ and $\left(M_{2}, g_{2}\right)$ be compact real analytic Riemannian manifolds, $n \geq 3$, with mutual boundary whose DN maps agree on an open set $\Gamma \subset \partial M$. Assume also that $\Gamma$ is real analytic. Then there is a diffeomorphic (global) harmonic morphism $J: M_{1}^{\Gamma} \rightarrow M_{2}^{\Gamma}$ such that $J$ is real analytic in $M_{1}^{\Gamma}$ and $\left.J\right|_{\Gamma}=I d$.

Proof We only need to show that $J$ is real analytic in $M_{1}^{\Gamma}$. In the interior this follows from the representation (9) in terms of harmonic coordinates, which are real analytic in the interior. Near points of $\Gamma$ this follows from the statement of Lemma 3.1, which implies that near points of $\Gamma$ one has $J=\psi_{2}^{-1} \circ \psi_{1}$ where $\psi_{j}$ are real analytic boundary normal coordinates. 


\section{Recovery of the Riemannian metric from a harmonic morphism}

In the previous section we showed that the Poisson embedding can be used to determine the manifold up to a global harmonic morphism from the knowledge of the DN map in the real analytic case, $n \geq 3$. To give a new proof for the Calderón problem in the real analytic case, we need to show that a global harmonic morphism in this case is an isometry. Throughout this section, we assume that $\left(M_{j}, g_{j}\right), j=1,2$, are compact connected and $C^{\infty}$ smooth, $n \geq 3$.

We show that if $J=P_{2}^{-1} \circ P_{1}: M_{1}^{\Gamma} \rightarrow M_{2}^{\Gamma}$ is defined, and thus is a global harmonic morphism, it is then a homothety,

$$
J^{*} g_{2}=\lambda g_{1}, \quad \lambda \text { constant }
$$

when $n \geq 3$. If we additionally assume that the DN-maps of $\left(M_{1}, g_{1}\right)$ and $\left(M_{2}, g_{2}\right)$ agree on $\Gamma \subset \partial M$, then boundary determination implies that $\lambda=1$.

It is known that a mapping between Riemannian manifolds having the same dimension $n \geq 3$ that pulls back local harmonic functions to local harmonic functions is in fact a homothety [13], see also [1, Cor. 3.5.2]. Our definition of harmonic morphisms assumes that the mapping pulls back global harmonic functions to global harmonic functions. Our condition is seemingly slightly different, but it follows from the next result that, for manifolds having the same dimension, these conditions are equivalent.

We give a proof that a global harmonic morphism is in fact a homothety when $n \geq 3$ by using harmonic coordinates. This seems to give a new proof for the result in the local case as well.

Proposition 4.1 Let $\left(M_{1}, g_{1}\right)$ and $\left(M_{2}, g_{2}\right)$ be $C^{\infty}$ Riemannian manifolds having the same dimension $n \geq 3$ and having a mutual boundary $\partial M$. Let $\Gamma \subset \partial M$ be a nonempty open set, and let $\bar{J}:\left(M_{1}^{\Gamma}, g_{1}\right) \rightarrow\left(M_{2}^{\Gamma}, g_{2}\right)$ be a locally diffeomorphic $C^{\infty}$ global harmonic morphism. Then $J$ is a homothety.

Proof Let $x \in M_{1}^{\Gamma}$ and let $U=\left(u_{f_{1}}^{2}, \ldots, u_{f_{n}}^{2}\right)$ be an $n$-tuple of global harmonic functions that define harmonic coordinates on $\Omega_{2}$ near $J(x) \in M_{2}^{\Gamma}$, where $f_{k} \in C_{c}^{\infty}(\Gamma)$. This can be done by Proposition 2.2 upon choosing $\left\{d u_{f_{1}}^{2}(J(x)), \ldots, d u_{f_{n}}^{2}(J(x))\right\}$ linearly independent. Since $J$ is locally invertible, we have that $V=J^{*} U$ is a coordinate system near $x$ on $\Omega_{1}:=J^{-1}\left(\Omega_{2}\right)$. Since $J$ is a global harmonic morphism, the coordinate system $V$ is harmonic.

In the coordinates $V$ and $U$ the coordinate representations of harmonic functions $u_{f}$ and $v_{f}=J^{*} u_{f}$ agree for arbitrary $f \in C_{c}^{\infty}(\Gamma)$. This is because

$$
u \circ U^{-1}=v \circ V^{-1} \Longleftrightarrow v=u \circ U^{-1} \circ V=u \circ J=J^{*} u .
$$

In any $g_{j}$-harmonic coordinates, $j=1,2$, the Laplace-Beltrami operator is particularly simple:

$$
\Delta_{g_{j}} u=-g_{j}^{a b} \partial_{a} \partial_{b} u, \quad j=1,2 .
$$


Here we abuse notation and denote by $g_{1}$ and $g_{2}$ the coordinate representations $\left(V^{-1}\right)^{*} g_{1}$ and $\left(U^{-1}\right)^{*} g_{2}$, respectively. Define $z:=V(x)=U(J(x))$ so $z \in \mathbb{R}^{n}$, and $\Omega:=V\left(\Omega_{1}\right)=U\left(\Omega_{2}\right) \subset \mathbb{R}^{n}$.

For any symmetric matrix $\left(H_{i j}\right)$ such that $g_{2}^{i j}(z) H_{i j}=0$ we may find by Runge approximation a global $g_{2}$-harmonic function $u=u_{f}$ with $f \in C_{c}^{\infty}(\Gamma)$ whose Hessian at $z$ is $\left(H_{i j}\right)$ in the $g_{2}$-harmonic coordinates $U$. This is proved in Proposition A.5 (note that $\operatorname{Hess}_{g}(w)$ corresponds to $\left(\partial_{j k} w\right)$ in harmonic coordinates). Thus we have that

$$
\operatorname{Tr}\left(g_{1}(z)^{-1} H\right)=0=\operatorname{Tr}\left(g_{2}(z)^{-1} H\right)
$$

since the coordinate representations of the harmonic functions $v=v_{f}$ and $u=u_{f}$, and thus their Hessians, agree in the coordinates $V$ and $U$.

Since $H$ can be any symmetric matrix with $g_{2}^{i j}(z) H_{i j}=0$, the above means that $g_{1}^{-1}$ and $g_{2}^{-1}$ have the same orthocomplement at $z$ with respect to the Hilbert-Schmidt inner product in the space of symmetric matrices. Thus $g_{1}^{-1}(z)=\lambda(z)^{-1} g_{2}^{-1}(z)$ for some nonzero real number $\lambda(z)$. Due to the positive definiteness of $g_{j}, j=1,2$, we have that $\lambda(z)>0$. The argument above can be repeated for all $z \in \Omega$, and we have

$$
g_{1}(z)=\lambda(z) g_{2}(z), \quad z \in \Omega
$$

where $\lambda$ is a positive smooth function ( $\lambda$ is smooth since $g_{1}$ and $g_{2}$ are).

We next show that the function $\lambda$ is constant in $\Omega$. For this we use the fact that the coordinates, where (18) holds, are harmonic. This is equivalent to saying that the contracted Christoffel symbols $g_{j}^{a b} \Gamma_{a b}^{c}\left(g_{j}\right)$ vanish. By lowering the index, this means that

$$
\Gamma_{a}\left(g_{j}\right)=0
$$

where $\Gamma_{a}\left(g_{j}\right)=-\left|g_{j}\right|^{-1 / 2}\left(g_{j}\right)_{a b} \partial_{c}\left(\left|g_{j}\right|^{1 / 2}\left(g_{j}\right)^{b c}\right)$. By taking the contracted Christoffel symbol of the Eq. 18, we have

$$
0=\Gamma_{a}\left(g_{1}\right)=\Gamma_{a}\left(\lambda g_{2}\right)=\Gamma_{a}\left(g_{2}\right)-\frac{n-2}{2} \partial_{a} \log \lambda=-\frac{n-2}{2} \partial_{a} \log \lambda
$$

Here we have used the following simple computation for the conformal scaling of the contracted Christoffel symbols:

$$
\begin{aligned}
\Gamma_{a}(\lambda g) & =-|\lambda g|^{-1 / 2}(\lambda g)_{a b} \partial_{c}\left(|\lambda g|^{1 / 2}(\lambda g)^{b c}\right) \\
& =-\lambda^{-n / 2+1}|g|^{-1 / 2} g_{a b} \partial_{c}\left(\lambda^{n / 2-1}|g|^{1 / 2} g b c\right. \\
& =\lambda^{-n / 2+1}\left(\lambda^{n / 2-1} \Gamma_{a}-\partial_{a} \lambda^{n / 2-1}\right) \\
& =\Gamma_{a}-\lambda^{-n / 2+1}\left(\frac{n}{2}-1\right) \lambda^{n / 2-2} \partial_{a} \lambda=\Gamma_{a}-\frac{n-2}{2} \partial_{a} \log \lambda
\end{aligned}
$$


Since $n \geq 3$, Eq. 19 shows that $\lambda$ is constant in $\Omega$. Recalling that $g_{1}(z)$ and $g_{2}(z)$ were the coordinate representations of $g_{1}$ and $g_{2}$ in coordinates $V$ and $U$, with $V=J^{*} U$, we have $g_{1}=\lambda J^{*} g_{2}$ on $\Omega_{1}$. Since this identity holds near an arbitrary point $x \in M_{1}^{\Gamma}$ and since $M_{1}^{\Gamma}$ is connected, we have proved the claim.

Remark 4.2 We remark that in the setting of the proof above we can by Eq. 17 actually express (a multiple of) $g_{j}^{-1}$ at $z$ in terms of Hessians of solutions $u_{f}^{j}$ at $z$ for some $f \in C_{c}^{\infty}(\Gamma), j=1,2$. Let $H_{j}^{k}, k=1, \ldots, m, m=\frac{n(n+1)}{2}-1$, be a basis for the orthocomplement $\left\{g_{j}(z)^{-1}\right\}^{\perp}$ in the space of symmetric matrices equipped with the Hilbert-Schmidt inner product. By the Runge approximation of Proposition A.5, we may find $f_{k}$ so that $H_{k}^{j}=\operatorname{Hess}_{g_{1}}\left(u_{f_{k}}^{1}(z)\right)=\operatorname{Hess}_{g_{2}}\left(u_{f_{k}}^{2}(z)\right)$.

Then we have

$$
g_{j}^{-1}(z)=\lambda_{j} *\left(H_{j}^{1} \wedge H_{j}^{2} \wedge \cdots \wedge H_{j}^{m}\right), \quad \lambda_{j}=\text { constant } \neq 0, j=1,2 .
$$

Here $*$ is the Hodge star operator in the space of symmetric matrices defined with respect to the Hilbert-Schmidt inner product and $\wedge$ is the wedge product in that space. Equation (20) holds because it follows from the definition of the Hodge star that the right hand side is orthogonal to each $H_{j}^{k}$. Thus the right hand side has the same orthocomplement as $g_{j}^{-1}\left(x_{0}\right)$ has.

Another remark is that since a homothety maps harmonic functions to harmonic functions, we have that a mapping between same dimensional Riemannian manifolds is global harmonic morphism if and only if it is a local harmonic morphism as defined in [1, Definition 4.1.1].

Next we show that if the DN maps agree, the homothety constant $\lambda$ is 1 .

Proposition 4.3 Assume the conditions of Proposition 4.1, and assume in addition that the DN maps of $\left(M_{1}, g_{1}\right)$ and $\left(M_{2}, g_{2}\right)$ agree on an open subset $\Gamma$ of the boundary. Also assume that $\left.J\right|_{\Gamma}=\mathrm{Id}$. Then

$$
J^{*} g_{2}=g_{1}
$$

Proof By Proposition 4.1, we know that $J$ is homothety and that

$$
g_{1}=\lambda J^{*} g_{2} \text { in } M_{1}^{\Gamma},\left.\quad J\right|_{\Gamma}=\mathrm{Id}
$$

Fix a point $p \in \Gamma$, and use boundary determination (see the proof of Lemma 3.1) to deduce that

$$
g_{1}=\Psi^{*} g_{2} \text { in } U_{1},\left.\quad \Psi\right|_{U_{1} \cap \Gamma}=\mathrm{Id}
$$

for some diffeomorphism $\Psi$ defined in a neighborhood $U_{1}$ of $p$ in $M_{1}^{\Gamma}$.

Now if $v \in T_{p}(\partial M)$, the first equation and the fact that $\left.J\right|_{\Gamma}=\operatorname{Id}$ imply that

$$
g_{1}(v, v)=\lambda g_{2}(v, v)
$$


while the second equation gives that

$$
g_{1}(v, v)=g_{2}(v, v)
$$

Thus $\lambda=1$.

Combining the results so far, we have achieved a new proof of the uniqueness in the Calderón problem in the real analytic case when $n \geq 3$ [28]:

Theorem Let $\left(M_{1}, g_{1}\right)$ and $\left(M_{2}, g_{2}\right)$ be compact real analytic Riemannian manifolds, $n \geq 3$, with mutual boundary whose DN maps agree on an open set $\Gamma \subset \partial M$. Assume also that $\Gamma$ is real analytic. Then there is a real analytic diffeomorphism $J: M_{1}^{\Gamma} \rightarrow M_{2}^{\Gamma}$ such that $g_{1}=J^{*} g_{2}$ and $\left.J\right|_{\Gamma}=I d$.

\section{Uniqueness in the 2D Calderón problem}

In this section we use the Poisson embedding technique to give a new proof of uniqueness in the Calderón problem in dimension 2. This result is also due to [28]. In this section we assume $\left(M_{1}, g_{1}\right)$ and $\left(M_{2}, g_{2}\right)$ are compact, connected $C^{\infty}$ Riemannian manifolds with mutual boundary $\partial M$. Note that it is not required that the manifolds are real analytic.

Theorem 5.1 (Uniqueness in the Calderón problem in 2D) Let $\left(M_{1}, g_{1}\right)$ and $\left(M_{2}, g_{2}\right)$ be two-dimensional compact connected $C^{\infty}$ Riemannian manifolds with mutual boundary д $M$. Assume that the DN maps agree on an open subset $\Gamma \subset \partial M$. Then there is a conformal diffeomorphism $J: M_{1}^{\Gamma} \rightarrow M_{2}^{\Gamma}$ such that

$$
J^{*} g_{2}=\lambda g_{1}
$$

Here $\lambda$ is a smooth positive function in $M_{1}^{\Gamma},\left.\lambda\right|_{\Gamma}=1$, and $\left.J\right|_{\Gamma}=\mathrm{Id}$.

The proof relies on the fact that on two-dimensional manifolds there exist isothermal coordinates near any point, i.e. coordinates $\left(u_{1}, u_{2}\right)$ such that $d u_{1}=* d u_{2}$, see [38, Section 5.10]. In these coordinates the metric looks like $g_{j k}=c \delta_{j k}$ for some positive function $c$. Isothermal coordinates are also harmonic coordinates in dimension 2 . We will use both of these facts.

We first prove local determination of harmonic functions near a boundary point, and then extend local determination to global determination. These are analogues of Lemma 3.2 and Theorem 3.3. After this, a two-dimensional version of Proposition 4.1 determines the metric up to a conformal mapping.

For the determination of harmonic functions near a boundary point, we note that in isothermal coordinates a $g$-harmonic function actually satisfies the Laplace equation in a subset $\mathbb{R}^{2}$. We show that the boundary determination result [30] of the metric in boundary normal coordinates implies determination of the metric on the boundary also in isothermal coordinates. Determination of harmonic functions near the boundary then follows from unique continuation for harmonic functions on $\mathbb{R}^{2}$. 
The determination of harmonic functions near the boundary in isothermal coordinates involves some technicalities. These are consequences of the fact that the boundary determination result of [30], that we rely on, is given in boundary normal coordinates instead of isothermal coordinates. We address the technicalities in the next lemma. The proof of the lemma follows from the usual construction of isothermal coordinates [38, Section 5.10] and by referring to the boundary determination result [30, p. 1106]. We omit the actual proof.

Lemma 5.2 Let $\left(M_{1}, g_{1}\right)$ and $\left(M_{2}, g_{2}\right)$ be two-dimensional Riemannian manifolds with boundary whose DN maps agree on an open subset $\Gamma \subset \partial M$. Then for any $p \in \Gamma$ there are isothermal coordinates $U_{j}, j=1,2$, defined on neighborhoods $\Omega_{j} \subset M_{j}^{\Gamma}$ of $p$ such that the following statements hold:

(1) There is an open subset $\Gamma_{0}$ of $\Gamma$ with $p \in \Gamma_{0}$ such that $U_{1}\left(\Gamma_{0}\right)=U_{2}\left(\Gamma_{0}\right)=: \tilde{\Gamma} \subset$ $\mathbb{R}^{2}$ and $\left.U_{1}\right|_{\Gamma_{0}}=\left.U_{2}\right|_{\Gamma_{0}}$.

(2) If $f \in C_{c}^{\infty}(\Gamma)$, then the Cauchy data of the coordinate representations $U_{1}^{-1 *} u_{f}^{1}$ and $U_{2}^{-1 *} u_{f}^{2}$ agree on $\tilde{\Gamma} \subset \mathbb{R}^{2}$.

Lemma 5.3 (Near boundary determination in 2D) Assume the conditions in the previous lemma. Then for any $p \in \Gamma$ there are isothermal coordinates $U_{j}, j=1,2$, defined on neighborhoods $\Omega_{j} \subset M_{j}^{\Gamma}$ of p such that for $f \in C_{c}^{\infty}(\Gamma)$, we have

$$
u_{f}^{1} \circ U_{1}^{-1}(x)=u_{f}^{2} \circ U_{2}^{-1}(x), \quad x \in U_{1}\left(\Omega_{1}\right) \cap U_{2}\left(\Omega_{2}\right) \subset\left\{x^{2} \geq 0\right\} .
$$

Moreover, there exists an open set $B \subset M_{1}^{\Gamma}$ with $\Gamma \subset B$ and $a C^{\infty}$ diffeomorphism $F: B \rightarrow F(B) \subset M_{2}^{\Gamma}$ such that

$$
F(B) \subset M_{2}^{\Gamma}, \quad P_{1}=P_{2} \circ F \text { on } B .
$$

Proof Let $f \in C_{c}^{\infty}(\Gamma)$ and let $u_{f}^{j}, j=1,2$, be the corresponding harmonic functions on $\left(M_{j}, g_{j}\right)$. Let $U_{j}$ be the isothermal coordinates of the previous lemma. Then the Cauchy data of $u_{f}^{j}$ agree on $\tilde{\Gamma} \subset \mathbb{R}^{2}$ in coordinates $U_{j}$. In isothermal coordinates, which are always also harmonic coordinates in dimension 2, the Laplace-Beltrami equation for $u_{f}^{j}$ reads

$$
c_{1}^{-1} \Delta_{\mathbb{R}^{n}}\left(u_{f}^{1} \circ U_{1}^{-1}\right)=0=c_{2}^{-1} \Delta_{\mathbb{R}^{n}}\left(u_{f}^{2} \circ U_{2}^{-1}\right) .
$$

Thus we see that $u_{f}^{j} \circ U_{j}^{-1}$ satisfy the same Euclidean Laplace equation with the same Cauchy data locally on a smooth mutual part of the boundary of the domain. By elliptic unique continuation, see e.g. [25, Theorem 3.3.1], and by setting $F=U_{2}^{-1} \circ U_{1}$ we obtain the claim with $B$ replaced by $U_{1}^{-1}\left(U_{1}\left(\Omega_{1}\right) \cap U_{2}\left(\Omega_{2}\right)\right)$. We can then enlarge $B$ as in Lemma 3.2 to conclude the proof.

We record the following: 
Proposition 5.4 Let $\left(M_{1}, g_{1}\right)$ and $\left(M_{2}, g_{2}\right)$ be two-dimensional $C^{\infty}$ Riemannian manifolds with mutual boundary. Let $J: M_{1}^{\Gamma} \rightarrow M_{2}^{\Gamma}$ be a locally diffeomorphic $C^{\infty}$ global harmonic morphism. Then $J$ is conformal,

$$
J^{*} g_{2}=\lambda g_{1} \text { in } M_{1}^{\Gamma}
$$

for some positive function $\lambda \in C^{\infty}\left(M_{1}^{\Gamma}\right)$.

The proof is identical to that of Proposition 4.1 except that we cannot deduce that $\lambda(x)$ is constant by the argument using Eq. (19). We omit the proof.

We will now prove global determination of harmonic functions.

Theorem 5.5 Let $\left(M_{1}, g_{1}\right)$ and $\left(M_{2}, g_{2}\right)$ be compact 2-dimensional $C^{\infty}$ smooth Riemannian manifolds with mutual boundary. Let $\Gamma$ be an open subset of $\partial M$. Assume that there is a neighborhood $B \subset M_{1}^{\Gamma}$ of a boundary point $p \in \Gamma$ and a mapping $F: B \rightarrow F(B) \subset M_{2}^{\Gamma}$ diffeomorphic onto its image such that

$$
P_{1}=P_{2} \circ F \text {. }
$$

Then we have $P_{1}\left(M_{1}^{\Gamma}\right)=P_{2}\left(M_{2}^{\Gamma}\right)$ and

$$
J=P_{2}^{-1} \circ P_{1}: M_{1}^{\Gamma} \rightarrow M_{2}^{\Gamma}
$$

is $C^{\infty}$ diffeomorphic global harmonic morphism extending $F$.

Proof We proceed as in the proof of Theorem 3.3 to which we refer the reader for more details. Let us recall the notation and some facts from there. We redefine $\emptyset \neq B \subset M_{1}^{\Gamma}$ to be the largest open connected set such that $P_{1}(B) \subset P_{2}\left(M_{2}^{\Gamma}\right)$. The task is to show that $B$ is closed. We argue by contradiction and assume that it is not. Then the points $x_{1} \in \partial B \backslash B$ and $x_{2} \in M_{2}^{\Gamma}$ are limits of sequences $\left(p_{k}\right) \subset B$ and $\left(J\left(p_{k}\right)\right) \subset J(B)$. If $f \in C_{c}^{\infty}(\Gamma)$, we have $u_{f}^{1}=u_{f}^{2} \circ J$ on $B$.

We construct isothermal coordinates on neighborhoods $\Omega_{1}$ of $x_{1} \in M_{1}^{\Gamma}$ and $\Omega_{2}$ of $x_{2} \in M_{2}^{\Gamma}$ as follows. Let us first choose by Runge approximation a boundary function $f \in C_{c}^{\infty}(\Gamma)$ such that

$$
d u_{f}^{2}\left(x_{2}\right) \neq 0
$$

Let us denote by $u_{1}$ a harmonic conjugate of $u_{f}^{2}$ near $x_{2}$. This is a function solving near $x_{2}$ the equation

$$
d u_{1}=-*_{g_{2}} d u_{f}^{2}
$$

where $*_{g_{2}}$ is the Hodge star of $g_{2}$. A local solution $u_{1}$ exists since the right hand side is closed, because $u_{f}^{2}$ is harmonic. We may assume $u_{1}\left(x_{2}\right)=0$. Let us denote

$$
U=\left(u_{1}, u_{f}^{2}\right)
$$


Then $U$ is an isothermal coordinate system on a neighborhood $\Omega_{2}$ of $x_{2}$. solves

Likewise, let $v_{1}$ be a harmonic conjugate of $u_{f}^{1}$ near $x_{1}$ with $v_{1}\left(x_{1}\right)=0$. Thus $v_{1}$

$$
d v_{1}=-*_{g_{1}} d u_{f}^{1}
$$

We set

$$
V=\left(v_{1}, u_{f}^{1}\right)
$$

These are isothermal coordinates on a neighborhood $\Omega_{1}$ of $x_{1}$. The fact that the Jacobian of $V$ is invertible at $x_{1}$ follows from

$$
d u_{f}^{1}\left(x_{1}\right)=\lim d u_{f}^{1}\left(p_{k}\right)=\lim d u_{f}^{2}\left(J\left(p_{k}\right)\right)=d u_{f}^{2}\left(x_{2}\right) \neq 0 .
$$

Redefine $\Omega_{1}$, if necessary, so that $V\left(\Omega_{1}\right) \subset U\left(\Omega_{2}\right)$.

We next show that on $B \cap \Omega_{1}$ we have $v_{1}=u_{1} \circ J$. By using $u_{f}^{1}=u_{f}^{2} \circ J$ on $B$ we have

$$
-d v_{1}=*_{g_{1}} d u_{f}^{1}=*_{g_{1}} d\left(J^{*} u_{f}^{2}\right)=*_{g_{1}} J^{*} d u_{f}^{2}
$$

By Lemma 2.4 $J$ is a $C^{\infty}$ diffeomorphism in $B$. By Proposition 5.4 applied with $M_{1}^{\Gamma}$ replaced by $B$ and $M_{2}^{\Gamma}$ replaced by $J(B)$ (the proof of Proposition 5.4 is really a pointwise argument and applies in this case), we have that $J$ is a conformal mapping on $B, J^{*} g_{2}=\lambda g_{1}$. Thus we have

$$
*_{g_{1}} J^{*} d u_{f}^{2}=J^{*}\left(*_{\left(g_{2} /\left(\lambda \circ J^{-1}\right)\right)} d u_{f}^{2}\right) .
$$

Since Hodge star is conformally invariant when operating on 1-forms in dimension 2 , the above is

$$
J^{*}\left(*_{g_{2}} d u_{f}^{2}\right)=-J^{*} d u_{1}=-d\left(u_{1} \circ J\right)
$$

Thus $d v_{1}=d\left(u_{1} \circ J\right)$ on $\Omega_{1} \cap B$. Since $v_{1}\left(x_{1}\right)=u_{1}\left(x_{2}\right)=0$, we have $v_{1}=u_{1} \circ J$ on $\Omega_{1} \cap B$. Consequently on $\Omega_{1} \cap B$ we have

$$
V=J^{*} U \text {, or equivalently } J=U^{-1} \circ V .
$$

(The point here is that $U^{-1} \circ V$ is defined on the whole $\Omega_{1}$ and gives us a good candidate for a local extension of $J$ onto the whole $\Omega_{1}$.)

Let $f \in C_{c}^{\infty}(\Gamma)$. To conclude the proof, we will show that on $V\left(\Omega_{1}\right)$, we have

$$
u_{f}^{1} \circ V^{-1}=u_{f}^{2} \circ U^{-1}
$$


Since $u_{f}^{1}=u_{f}^{2} \circ J$ on $B$, for $f \in C_{c}^{\infty}(\Gamma)$, the above holds on the open set $V\left(\Omega_{1} \cap B\right)$. Since the coordinates in question are isothermal and harmonic (where $\Gamma^{a}\left(g_{j}\right)=0$ ) we have that

$$
c_{1}^{-1} \Delta_{\mathbb{R}^{2}}\left(u_{f}^{1} \circ V^{-1}\right)=0=c_{2}^{-1} \Delta_{\mathbb{R}^{2}}\left(u_{f}^{2} \circ U^{-1}\right) .
$$

Thus $u_{f}^{1} \circ V^{-1}$ and $u_{f}^{2} \circ U^{-1}$ both satisfy the Laplace equation in $V\left(\Omega_{1}\right) \subset \mathbb{R}^{2}$. Since these functions agree on the open set $V\left(\Omega_{1} \cap B\right)$ they agree everywhere on $V\left(\Omega_{1}\right)$ by unique continuation. This shows that we may indeed extend $J$ to $B \cup \Omega_{1}$, which gives a contradiction and concludes the proof.

Proof of Theorem 5.1 By Lemma 5.3, we have that there is $B \subset M_{1}^{\Gamma}$ and a diffeomorphic harmonic morphism $F: B \rightarrow F(B) \subset M_{2}^{\Gamma}$. By Theorem 5.5 the mapping $F$ extends to a global harmonic morphism $J: M_{1}^{\Gamma} \rightarrow M_{2}^{\Gamma}$. Proposition 5.4 shows that $J$ is a conformal mapping. That the implied conformal factor is 1 on $\Gamma$ follows from calculations in the proof of Proposition 4.3.

\section{On determining the coefficients of quasilinear elliptic operators from source-to-solution mapping}

In this section we apply the Poisson embedding technique for a Calderón type inverse problem for second order quasilinear elliptic operators on Riemannian manifolds. Let $(M, g)$ be a Riemannian manifold with boundary. The quasilinear operators

$$
Q: C^{\infty}(M) \rightarrow C^{\infty}(M)
$$

we study are assumed to have the coordinates representation

$$
Q u(x)=\mathcal{A}^{a b}(x, u(x), d u(x)) \nabla_{a} \nabla_{b} u(x)+\mathcal{B}(x, u(x), d u(x)) .
$$

Here we assume that $\mathcal{A}^{a b}(x, c, \sigma) \in T_{x}^{2} M$ for given $(x, c, \sigma) \in M \times \mathbb{R} \times T_{x}^{*} M$ is a 2-tensor field and $\mathcal{B}(x, c, \sigma)$ is a function. The covariant derivative $\nabla$ is determined by $g$ as usual. We consider $\mathcal{A}$ and $\mathcal{B}$ as mappings

$$
\mathcal{A}: M \times \mathbb{R} \otimes T^{*} M \rightarrow T_{0}^{2}(M)
$$

and

$$
\mathcal{B}: M \times \mathbb{R} \otimes T^{*} M \rightarrow \mathbb{R}
$$

where $\pi(\mathcal{A}(x, c, \sigma))=x$. Here $\pi$ is the canonical projection $T_{0}^{2}(M) \rightarrow M$ for 2-contravariant tensors. The notation $M \times \mathbb{R} \otimes T^{*} M$ refers to the subset

$$
\left\{(x, c, \sigma) \in M \times \mathbb{R} \times T_{x}^{*} M\right\}
$$


of $M \times \mathbb{R} \times T^{*} M$. We can also think of $M \times \mathbb{R} \otimes T^{*} M$ as tensor product of the trivial line bundle $M \times \mathbb{R}$ and the vector bundle $T^{*} M$.

We assume that $Q$ is quasilinear elliptic, which means that for all $(x, c, \sigma) \in$ $M \times \mathbb{R} \otimes T^{*} M$ and $\xi \in T_{x}^{*} M$ we have

$$
\mathcal{A}^{a b}(x, c, \sigma) \xi_{a} \xi_{b} \geq \lambda \mid \xi_{g}^{2}, \quad \lambda>0
$$

We assume that the coefficients $\mathcal{A}$ and $\mathcal{B}$ are $C^{\infty}$ smooth and in the main theorem of this section, Theorem 6.2, we assume that they are real analytic. We will assume throughout that 0 is a solution, i.e. $Q(u)=0$, which is equivalent with the condition

$$
\mathcal{B}(x, 0,0)=0
$$

The linearization of $Q$ at $u=0$ is the operator

$$
L u=\mathcal{A}^{a b}(x, 0,0) \nabla_{a} \nabla_{b} u+\frac{\partial \mathcal{B}}{\partial u}(x, 0,0) u+\frac{\partial \mathcal{B}}{\partial \sigma_{j}}(x, 0,0) \partial_{j} u
$$

where in the last term we have identified $T_{x}^{*} M$ with $\mathbb{R}^{n}$. We will also assume that, for some fixed $\alpha$ with $0<\alpha<1$,

$$
L: C^{2, \alpha}(M) \cap H_{0}^{1}(M) \rightarrow C^{\alpha}(M) \text { is invertible. }
$$

It follows from these assumptions and from the implicit function theorem in Banach spaces that there are unique small solutions of $Q(u)=f,\left.u\right|_{\partial M}=0$ when $f$ is small. We record these facts in the following lemma. We omit the proof.

Proposition 6.1 Let $(M, g)$ be a compact manifold with smooth boundary, let $\mathcal{A}, \mathcal{B}$ be $C^{\infty}$ maps satisfying (22)-(25), and let

$$
Q(u)=\mathcal{A}^{a b}(x, u, d u) \nabla_{a} \nabla_{b} u+\mathcal{B}(x, u, d u) .
$$

Assume that $L$ is the linearization of $Q$ at $u=0$ given in (26), and assume that (27) holds.

There are constants $C, \varepsilon, \delta>0$ such that whenever $\|f\|_{C^{\alpha}(M)} \leq \varepsilon$, the equation $Q(u)=f$ in $M$ with $\left.u\right|_{\partial M}=0$ has a solution $u \in C^{2, \alpha}(M)$ satisfying $\|u\|_{C^{2, \alpha}(M)} \leq$ $C\|f\|_{C^{\alpha}(M)}$. If $u_{j} \in C^{2, \alpha}(M), j=1,2$, both solve $Q\left(u_{j}\right)=f$ in $M$ with $\left.u_{j}\right|_{\partial M}=0$ and $\left\|u_{j}\right\|_{C^{2, \alpha}(M)} \leq \delta$, then $u_{1}=u_{2}$.

Operators of the above form appear e.g. in the study of minimal surfaces or prescribed scalar curvature questions (Yamabe problem), see [14,38] for more information.

We describe the inverse problem we are about to study. Let $Q, \varepsilon, \delta$ be as in Proposition 6.1. We assume that we know the source-to-solution mapping of $Q$ on an open subset $W$ of $M$. The source-to-solution mapping

$$
S:\left\{f \in C_{c}^{\infty}(W) ;\|f\|_{C^{\alpha}(M)} \leq \varepsilon\right\} \rightarrow C^{\infty}(W)
$$


is defined as

$$
S:\left.f \mapsto u\right|_{W},
$$

where $u$ is the unique solution to

$$
Q u=f \text { on } M, \quad u=0 \text { on } \partial M, \quad\|u\|_{C^{2, \alpha}(M)} \leq \delta .
$$

Note that indeed $u \in C^{\infty}(M)$, using Schauder estimates for linear elliptic equations and the fact that $u \in C^{2, \alpha}(M)$. The aim of the inverse problem is to determine the coefficients $\mathcal{A}$ and $\mathcal{B}$ of $Q$ up to a diffeomorphism and possible other symmetries of the problem. When the coefficients of $Q$ are real analytic, our main theorem shows that in this case there is only one additional symmetry, which we describe next.

We note that there is a simple transformation between coefficient $\mathcal{B}$ and the Christoffel symbols $\Gamma_{a b}^{k}$ contracted by $\mathcal{A}^{a b}$ that leaves the source-to-solution mapping intact: Assume that $u$ solves

$$
Q u=f \text { on } M \text {. }
$$

Then $u$ also solves

$$
\tilde{Q} u=f,
$$

where

$$
\tilde{Q} u=\mathcal{A}^{a b}(x, u(x), d u(x)) \tilde{\nabla}_{a} \tilde{\nabla}_{b} u+\tilde{\mathcal{B}}(x, u(x), d u(x))
$$

and where $\tilde{\mathcal{B}}$ is defined as

$$
\tilde{\mathcal{B}}(x, c, \sigma)=\mathcal{B}(x, c, \sigma)+\mathcal{A}(x, c, \sigma)^{a b}\left(\tilde{\Gamma}_{a b}^{k}(x)-\Gamma_{a b}^{k}(x)\right) \sigma_{k}
$$

Here $\tilde{\nabla}$ and $\tilde{\Gamma}_{a b}^{k}$ denote the Levi-Civita connection and Christoffel symbols of some other Riemannian metric $\tilde{g}$ on $M$. Therefore the source-to-solution mapping defined with respect to $\tilde{Q}$ coincides with the source-to-solution map $S$ of $Q$. Note that even though Christoffel symbols does not constitute a tensor field, the difference of two Christoffel symbols $\tilde{\Gamma}_{a b}^{k}-\Gamma_{a b}^{k}$ is a tensor field. It follows that we can not make the symmetry vanish by choosing a suitable coordinate system. This symmetry will be called the gauge symmetry of the inverse problem.

The gauge symmetry is an obstruction for finding $\mathcal{B}, \mathcal{A}$ and $\Gamma_{a b}^{k}$ independently of each other in the general case. However, in some cases when we have extra information about the coefficients $\mathcal{A}$ and $\mathcal{B}$, the gauge symmetry vanishes. We give examples of conditions when this happens in Corollary 6.3.

We remark that if the coefficients are not real analytic, other symmetries in the inverse problem can appear. An easy example is the standard Laplace-Beltrami operator in dimension 2 where one can scale the metric by a positive function that is 
constant 1 on the measurement set $W$ without affecting the source-to-solution mapping. Another similar example is given by the conformal Laplacian in dimensions $n \geq 3$ [26].

Our main theorem of this section is the following determination result.

Theorem 6.2 Let $\left(M_{1}, g_{1}\right)$ and $\left(M_{2}, g_{2}\right)$ be compact connected real analytic Riemannian manifolds with mutual boundary and assume that $Q_{j}, j=1,2$, are quasilinear operators of the form (21) having coefficients $\mathcal{A}_{j}, \mathcal{B}_{j}$ satisfying (22)-(27). Moreover, assume that $\mathcal{A}_{j}$ and $\mathcal{B}_{j}$ are real analytic in all their arguments.

Let $W_{j}, j=1,2$, be open subsets of $M_{j}$, and assume that there is a diffeomorphism $\phi: W_{1} \rightarrow W_{2}$ so that the source-to-solutions maps $S_{j}$ for $Q_{j}$ agree in the sense that

$$
\phi^{*} S_{2} f=S_{1} \phi^{*} f
$$

for all $f \in C_{c}^{\infty}\left(W_{2}\right)$ with $\|f\|_{C^{\alpha}\left(M_{2}\right)}$ sufficiently small.

Then there is a real analytic diffeomorphism $J: M_{1}^{\text {int }} \rightarrow M_{2}^{\text {int }}$ such that

$$
\mathcal{A}_{1}=J^{*} \mathcal{A}_{2}=: \mathcal{A}
$$

and

$$
\mathcal{B}_{1}-J^{*} \mathcal{B}_{2}=\mathcal{A}^{a b}\left(\Gamma\left(g_{1}\right)_{a b}^{k}-\Gamma\left(J^{*} g_{2}\right)_{a b}^{k}\right) \sigma_{k}
$$

The mapping $J$ satisfies

$$
\left.J\right|_{W_{1}^{\text {int }}}=\phi: W_{1}^{\text {int }} \rightarrow W_{2}^{\text {int }}
$$

where $W_{1}^{\text {int }}=W_{1} \cap M_{1}^{\text {int }}$ and $W_{2}^{\text {int }}=W_{2} \cap M_{2}^{\text {int }}$.

The assumption $\phi^{*} S_{2} f=S_{1} \phi^{*} f$, for $f \in C_{c}^{\infty}\left(W_{2}\right)$ small, means that the diagram

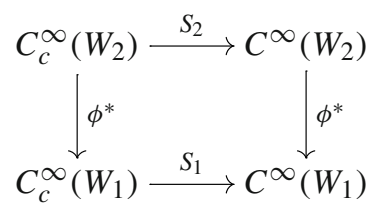

commutes when $f$ is small.

We describe our strategy for proving the theorem. By the arguments in the preceding sections and by the fact that solutions to quasilinear equations with real analytic coefficients are real analytic [33], it would be natural to define a mapping analogous to the Poisson embedding for the quasilinear elliptic operator $Q$ and then use tools analogous to those we built around the Poisson embedding. However, as far as we know, Runge approximation for quasilinear operators is not known. This prevents us of using this natural approach for the moment.

Instead we do the following. We linearize the source-to-solution mapping (at the mutual solution 0 ) that yields a linear Calderón type inverse problem for a linear second 
order elliptic operator whose source-to-solution map is known. For this linearized problem we use the Poisson embedding technique modified slightly to deal with the source-to-solution map instead of the DN map. In this way we will find the manifold up to a real analytic diffeomorphism. This is the first step. The modified Poisson embedding is given in Definition 6.1.

The second step is the following. We will see that knowing the source-to-solution map on the open set $W$ determines the coefficients $\mathcal{A}$ and $\mathcal{B}$ on $W$, up to the gauge symmetry described in (32). In this step we read the coefficients $\mathcal{A}$ and $\mathcal{B}$ in $W$ from the solutions, which is similar to the argument in Proposition 4.1.

Since we have determined the manifold up to a real analytic diffeomorphism, we can view the coefficients $\mathcal{A}$ and $\mathcal{B}$ on a single fixed manifold and use standard real analytic unique continuation there. This determines the coefficients of the quasilinear operator on the whole manifold up to a diffeomorphism and the gauge symmetry.

As already mentioned, with some suitable extra information about the coefficients $\mathcal{B}$ and $\mathcal{A}$ the gauge symmetry vanishes and we can determine $\mathcal{A}$ and $\mathcal{B}$ independently.

Corollary 6.3 Assume the conditions and notation in Theorem 6.2, and assume also one of the following:

(1) $\mathcal{A}_{1}(x, c, \sigma)\left(\right.$ or $\left.\mathcal{A}_{2}(x, c, \sigma)\right)$ is $s$-homogeneous in the $\sigma$-variable and $\mathcal{B}_{1}(x, c, \sigma)$ and $\mathcal{B}_{2}(x, c, \sigma)$ are $s^{\prime}$-homogeneous with $s^{\prime} \neq s+1$ for all $x \in W_{1}$ and $c \in \mathbb{R}$; or

(2) $\phi:\left(W_{1}^{\text {int }},\left.g_{1}\right|_{W_{1}^{\text {int }}}\right) \rightarrow\left(W_{2}^{\text {int }},\left.g_{2}\right|_{W_{2}^{\text {int }}}\right)$ is an isometry.

Then we have

$$
\mathcal{A}_{1}=J^{*} \mathcal{A}_{2} \text { and } \mathcal{B}_{1}=J^{*} \mathcal{B}_{2}
$$

and also

$$
\mathcal{A}_{1}^{a b} \Gamma_{a b}^{k}\left(g_{1}\right)=\left(J^{*} \mathcal{A}_{2}\right)^{a b} \Gamma_{a b}^{k}\left(J^{*} g_{2}\right)
$$

Note that the corollary does not claim that we can find the Riemannian metrics $g_{1}$ and $g_{2}$ up to $J$. An example satisfying the first condition is the nonlinear Schrödinger operator

$$
u \in C^{\infty} \mapsto-\Delta_{g} u+q|u|^{2} u, \quad q \in C^{\infty} .
$$

An example satisfying the second condition is the case, where one knows the Riemannian metric and the manifold $(\phi=\mathrm{Id})$ on a measurement set $W$.

\subsection{Linearized problem}

Let us first linearize the source-to-solution map of the quasilinear problem. This yields a Calderón type inverse problem for the linearized equation. We record the following result. We leave out the proof of the result and refer to [22] for a proof of a similar result. 
Proposition 6.4 Let $(M, g)$ and $Q$ be as in Proposition 6.1. Let $W \subset M$ be open, and let $S$ be the source-to-solution map defined in (29). Then, for any $f \in C_{c}^{\infty}(W)$,

$$
\lim _{t \rightarrow 0} \frac{S(t f)-S(0)}{t}=S^{L}(f) \quad\left(\text { limit in } C^{1}(\bar{W})\right)
$$

where $S^{L}: C_{c}^{\infty}(W) \rightarrow C^{\infty}(W), f \mapsto u$ is the source-to-solution map of the linearized equation $L u=f$ in $M$ with $\left.u\right|_{\partial M}=0$, where $L$ is given in (26).

We have now seen that linearizing the source-to-solution mapping $S$ of a quasilinear equation leads to a Calderón type inverse problem for a linear equation. Next we show that in the real analytic case the source-to-solution mapping of the linearized problem determines the manifold up to a real analytic diffeomorphism. Precisely we will prove:

Theorem 6.5 Let $\left(M_{1}, g_{1}\right)$ and $\left(M_{2}, g_{2}\right)$ be compact real analytic manifolds with mutual boundary. Let $L_{1}$ and $L_{2}$ be second order uniformly elliptic partial differential operators on $M_{1}$ and $M_{2}$, respectively, of the general form

$$
L_{j}=A_{j}^{a b}(x) \nabla_{a} \nabla_{b} u(x)+B_{j}^{a}(x) \nabla_{a} u(x)+C_{j}(x) u(x),
$$

where $A_{j}^{a b}$ and $B_{j}^{a}$ are the components of a 2-tensor field $A$ and vector field $B_{j}$, and $C$ is a function. Assume that $L_{j}$ are injective on $C^{\infty}\left(M_{j}\right) \cap H_{0}^{1}\left(M_{j}\right)$, and assume that $A, B$ and $C$ are real analytic up to boundary.

Let $W_{j} \subset M_{j}$ be open sets, and let $\phi: W_{1} \rightarrow W_{2}$ be a diffeomorphism so the source-to-solutions maps $S_{j}^{L}$ of $L_{j}$ satisfy

$$
S_{1}^{L} \phi^{*} f=\phi^{*} S_{2}^{L} f, \quad f \in C_{c}^{\infty}\left(W_{2}\right) .
$$

Then there is a real analytic diffeomorphism $J: M_{1}^{\mathrm{int}} \rightarrow M_{2}^{\mathrm{int}}$ with $\left.J\right|_{W_{1}^{\mathrm{int}}}=\left.\phi\right|_{W_{1}^{\mathrm{int}}}$ : $W_{1}^{\text {int }} \rightarrow W_{2}^{\text {int }}$. If $f \in C_{c}^{\infty}\left(W_{2}\right)$, then $u_{f \circ \phi}^{1}=J^{*} u_{f}^{2}$.

In the theorem $u_{f \circ \phi}^{1}$ and $u_{f}^{2}$ are the solutions to $L_{1} u_{f \circ \phi}^{1}=f \circ \phi$ in $M_{1}$ and to $L_{2} u_{f}^{2}=f$ in $M_{2}$ with $\left.u_{f \circ \phi}^{1}\right|_{\partial M}=\left.u_{f}^{2}\right|_{\partial M}=0$.

We prove the theorem by modifying the Poisson embedding technique to suit the study of source-to-solution map instead of the DN map. The arguments are very similar to those that we have used in the previous sections. We keep the exposition short.

Definition 6.1 (Poisson embedding for $L$ ) Let $(M, g)$ be a compact Riemannian manifold with boundary, and let $W$ be an open subset of $M$. Let $L$ be a second order elliptic differential operator of the form (33) which is injective on $C^{\infty}(M) \cap H_{0}^{1}(M)$. The Poisson embedding $R$ of the manifold $M$ is defined to be the mapping

$$
R: M^{\text {int }} \rightarrow \mathcal{D}^{\prime}(W)
$$


such that $R(x) f=u_{f}(x)$, where $u_{f}$ solves the Poisson problem

$$
\begin{aligned}
L u_{f} & =f \text { in } M, \\
u_{f} & =0 \text { on } \partial M .
\end{aligned}
$$

In the definition $\mathcal{D}^{\prime}(W)$ means $\left[C_{c}^{\infty}(W)\right]^{*}$ as usual. The reason why we consider $R$ to be defined only in the interior of $M$ is because we assume that the boundary values of the solutions $u_{f}$ vanish on the boundary. Thus we have no control on the points on the boundary by using solutions $u_{f}$. Even though $R$ is not the Poisson embedding of the previous section, we use the same name for $R$, and we note that $R$ is related to the linear elliptic operator $L$.

The basic properties of the Poisson embedding $R$ are as follows.

Proposition 6.6 Let $(M, g)$ be smooth compact manifold with boundary. For any $x \in$ $M^{\text {int }}$, one has $R(x) \in H^{-s}(W)$ whenever $s+2>n / 2$. The mapping $R$ is continuous $M^{\mathrm{int}} \rightarrow H^{-s-1}(W)$ and $k$ times Fréchet differentiable considered as a mapping $M^{\text {int }} \rightarrow H^{-s-1-k}(W)$. In particular, $R: M^{\text {int }} \rightarrow \mathcal{D}^{\prime}(W)$ is $C^{\infty}$ smooth in the Fréchet sense. The mapping $R$ can be extended continuously to a mapping $M \rightarrow H^{-s}(W)$ by defining $\left.R\right|_{\partial M}=0$.

The Fréchet derivative of $R$ at $x \in M^{\mathrm{int}}$ is a linear mapping given by

$$
D R_{x}: T_{x} M^{\mathrm{int}} \rightarrow \mathcal{D}^{\prime}(W), \quad\left(D R_{x} V\right) f=d u_{f}(x) \cdot V,
$$

where $u_{f}$ solves $L u_{f}=f$ in $M,\left.u_{f}\right|_{\partial M}=0, f \in C_{c}^{\infty}(W)$, and $\cdot$ refers to the canonical pairing of vectors and covectors on $M$.

In the statement, we are not claiming that the continuation of $R$ onto $M$ is injective on $\partial M$. We omit the proof of Proposition 6.6.

To prove that $R$ is an embedding, we use the following analogue of Proposition 2.2 which follows from a suitable Runge approximation result. Its proof is in Appendix A.

Proposition 6.7 Let $(M, g)$ be a compact manifold with boundary, and let L be a second order uniformly elliptic differential operator on $M$ which is injective on $C^{\infty}(M) \cap H_{0}^{1}(M)$. Let $W$ be a nonempty open subset of $M$, and denote by $u_{f}$ the solution of $L u=f$ in $M$ with $\left.u\right|_{\partial M}=0$.

(a) If $x \in M^{\text {int },} y \in M$ and $x \neq y$, there is $f \in C_{c}^{\infty}(W)$ such that

$$
u_{f}(x) \neq u_{f}(y)
$$

(b) If $x \in M^{\mathrm{int}}$ and $v \in T_{x}^{*} M$, there is $f \in C_{c}^{\infty}(W)$ such that

$$
d u_{f}(x)=v .
$$

Proposition 6.8 ( $R$ is an embedding) Let $M, L, R$ be as in Definition 6.1. The mapping $R: M^{\mathrm{int}} \rightarrow \mathcal{D}^{\prime}(W)$ is a $C^{\infty}$ embedding (and a $C^{k}$ embedding $M^{\mathrm{int}} \rightarrow H^{-s-1-k}(W)$ ) in the sense that it is injective with injective Fréchet differential on $T M^{\mathrm{int}}$. 
Proof The injectivity of $R$ follows from Proposition 6.7(a). Let $x \in M^{\text {int }}$ and $V \in$ $T_{x} M$. Assume that $0=\left(D R_{x} V\right) f=d u_{f}(x) \cdot V$ for all $f \in C_{c}^{\infty}(W)$. By Proposition 6.7(b) one can find $f \in C_{c}^{\infty}(W)$ so that $d u_{f}(x) \cdot V \neq 0$, unless $V=0$. This shows injectivity of the differential.

We construct next local coordinate systems from solutions $u_{f}$ to $L u_{f}=f,\left.u_{f}\right|_{\partial M}=$ 0 . We call these coordinates solution coordinates. These coordinates are constructed by Runge approximating local solutions to $L u=0$.

Lemma 6.9 (Solution coordinates) Let $(M, g)$ be a compact Riemannian manifold with boundary. Let $W$ be an open subset of $M$ and let $x_{0} \in M^{\text {int }}$. Then there is $C^{\infty}$ coordinate system on a neighborhood $\Omega$ of $x_{0}$ of the form $\left(u_{f_{1}}, \ldots, u_{f_{n}}\right)$ where each of the coordinate functions satisfies

$$
L u_{f_{j}}=f_{j} \text { in } M, \quad u_{f_{j}}=0 \text { on } \partial M,
$$

with $f_{j} \in C_{c}^{\infty}(W)$, and where

$$
f_{j}=0 \text { on } \Omega, \quad j=1, \ldots, n \text {. }
$$

If the coefficients of $L$ are real analytic, then $\left(u_{f_{1}}, \ldots, u_{f_{n}}\right)$ is real analytic on $\Omega$.

Proof Let $x_{0} \in M^{\text {int }}$. If $x_{0} \in W$, we redefine $W$ as a smaller open set such that $x_{0} \notin W$. By Proposition 6.7(b) we may find $f_{1}, \ldots, f_{n} \in C_{c}^{\infty}(W)$ such that the Jacobian matrix of $U=\left(u_{f_{1}}, \ldots, u_{f_{n}}\right)$ is the identity matrix at $x_{0}$. Thus $U$ is a coordinate system in some neighborhood $\Omega$ of $x_{0}$, and by shrinking $\Omega$ if necessary we may assume that $f_{j}=0$ in $\Omega$.

If the coefficients of $L$ are real analytic, the coordinate system $U$ is real analytic on $\Omega$ by elliptic regularity, since $\left.f_{j}\right|_{\Omega}=0$.

The next lemma is an analogue of Lemma 2.4, where we considered the composition of Poisson embeddings. If $W_{1}$ and $W_{2}$ are open subsets of $M_{1}$ and $M_{2}$ and $\phi: W_{1} \rightarrow$ $W_{2}$ is a diffeomorphism, we define a mapping $\phi^{*} R_{2}: M_{2}^{\text {int }} \rightarrow \mathcal{D}^{\prime}\left(W_{1}\right)$ by

$$
\left[\phi^{*} R_{2}\right](x) f=R_{2}(x)\left(f \circ \phi^{-1}\right),
$$

with $x \in M_{2}^{\text {int }}$ and $f \in C_{c}^{\infty}\left(W_{1}\right)$. From Propositions 6.6 and 6.8, it follows that $\phi^{*} R_{2}$ is a $C^{k}$ embedding $M_{2}^{\text {int }} \rightarrow H^{-s-1-k}(W), k=0,1, \ldots$

Lemma 6.10 Let $\left(M_{1}, g_{1}\right)$ and $\left(M_{2}, g_{2}\right)$ be compact manifolds with mutual boundary ә $M$. Let $W_{j} \subset M_{j}, j=1,2$, be open subsets and let $\phi$ be a diffeomorphism $W_{1} \rightarrow$ $W_{2}$. Assume that for some open set $B \subset M_{1}^{\text {int }}$

$$
R_{1}(B) \subset\left(\phi^{*} R_{2}\right)\left(M_{2}^{\text {int }}\right) .
$$

Then

$$
J=\left(\phi^{*} R_{2}\right)^{-1} \circ R_{1}
$$

is $C^{\infty}$ diffeomorphism $B \rightarrow J(B) \subset M_{2}^{\text {int }}$. 
Proof We prove the continuity of $J$ differently than we did in the corresponding situation in Lemma (2.4). It follows from Proposition 6.6 that we may continue $\phi^{*} R_{2}$ by zero to a continuous mapping $M_{2} \rightarrow H^{-s-1}\left(W_{1}\right)$. Let $E \subset M_{2}^{\text {int }}$ be closed in $M_{2}$. It follows that $\phi^{*} R_{2}(E)$ is closed in $H^{-s-1}\left(W_{1}\right)$ by continuity and by compactness of $M_{2}$. Since $\phi^{*} R_{2}$ is injective on $M_{2}^{\text {int }}$, we have that $I:=\left(\phi^{*} R_{2}\right)^{-1}$ is defined as a mapping $\phi^{*} R_{2}\left(M_{2}^{\text {int }}\right) \rightarrow M_{2}^{\text {int }}$ and we have that $I^{-1}(E)$ equals $\phi^{*} R_{2}(E)$, which is closed. Thus $I$ is continuous and consequently $J$ is continuous $B \rightarrow J(B)$ by Proposition 6.6.

Let $x_{0} \in M_{1}^{\text {int }}$ and let $U=\left(u_{f_{1}}^{2}, \ldots, u_{f_{n}}^{2}\right)$ be solution coordinates of Lemma 6.9 on a neighborhood $\Omega_{2}$ of $J\left(x_{0}\right)$, where $f_{k} \in C_{c}^{\infty}\left(W_{2}\right), k=1, \ldots, n$. In case $x_{0} \in W_{1}$, we may assume that $\phi\left(x_{0}\right) \notin \operatorname{supp}\left(f_{k}\right)$. We define $V:=J^{*} U$. Since $U$ is invertible, we have that $J=U^{-1} \circ V$. Since $J$ is continuous, the domain $\Omega_{1}:=J^{-1}\left(\Omega_{2}\right)$ of $V$ is an open neighborhood of $x_{0}$. We have $V=\left(v_{f_{1}}, \ldots, v_{f_{n}}\right)$ where $v_{f_{k}}$ satisfies

$$
\begin{aligned}
v_{f_{k}}(x) & =J^{*} u_{f_{k}}^{2}(x)=J^{*}\left(R_{2}(x) f_{k}\right)=R_{2}(J(x))\left(\left(f_{k} \circ \phi\right) \circ \phi^{-1}\right) \\
& =\left[\phi^{*} R_{2}\right](J(x))\left(f_{k} \circ \phi\right)=R_{1}(x)\left(f_{k} \circ \phi\right)=u_{f_{k} \circ \phi}^{1}(x) .
\end{aligned}
$$

Since $\phi\left(x_{0}\right) \notin \overline{\operatorname{supp}\left(f_{k}\right)}$, we have that each $u_{f_{k} \circ \phi}^{1}$ is $C^{\infty}$ near $x_{0}$. Thus $V$ is $C^{\infty}$ and consequently $J=U^{-1} \circ V$ is $C^{\infty}$. If $f \in \mathcal{D}^{\prime}\left(W_{1}\right)$, we have that

$$
u_{f}^{1}(x)=R_{1}(x) f=\left(\phi^{*} R_{2} \circ J\right)(x) f=\left[\phi^{*} R_{2}\right](J(x)) f=u_{f \circ \phi^{-1}}^{2}(J(x)),
$$

and

$$
d u_{f}^{1}\left(x_{0}\right) \cdot X=d\left(u_{f \circ \phi^{-1}}^{2} \circ J\right)\left(x_{0}\right) \cdot X=d u_{f \circ \phi^{-1}}^{2} \mid J\left(x_{0}\right) \cdot\left(D J\left(x_{0}\right)\right) X .
$$

It follows from Proposition 6.7 that $D J\left(x_{0}\right)$ is invertible. Since $J$ is also injective by Proposition 6.8, it follows that $J: B \rightarrow J(B)$ is $C^{\infty}$ diffeomorphism.

We prove next the main result of this subsection.

Proof of Theorem 6.5 Assume that the source-to-solutions maps agree:

$$
S_{1}^{L} \phi^{*} f=\phi^{*} S_{2}^{L} f, \quad f \in C_{c}^{\infty}\left(W_{2}\right) .
$$

By shrinking $W_{j}$ if necessary, we may assume that $W_{j}$ are small geodesic balls.

Redefine $B$ to be the largest connected open set of $M_{1}^{\text {int }}$ such that $R_{1}(B) \subset$ $\left(\phi^{*} R_{2}\right)\left(M_{2}^{\text {int }}\right)$. We have $W_{1}^{\text {int }} \subset B$. To see this, let $f \in C_{c}^{\infty}\left(W_{1}\right)$ and $x \in W_{1}^{\text {int }}$. We have by using definitions and (35) that

$$
\begin{aligned}
R_{1}(x) f & =u_{f}^{1}(x)=\left(S_{1}^{L} f\right)(x)=\left[S_{1}^{L}\left(\phi^{*}\left(f \circ \phi^{-1}\right)\right)\right](x)=\left[\phi^{*} S_{2}^{L}\left(f \circ \phi^{-1}\right)\right](x) \\
& =\left[S_{2}^{L}\left(f \circ \phi^{-1}\right)\right](\phi(x))=u_{f \circ \phi^{-1}}^{2}(\phi(x))=R_{2}(\phi(x))\left(f \circ \phi^{-1}\right) \\
& =\left[\left(\phi^{*} R_{2}\right)(\phi(x))\right] f .
\end{aligned}
$$


Note that $\phi(x) \in M_{2}^{\text {int }}$ since otherwise we have $u_{f}^{1}(x)=u_{f \circ \phi^{-1}}^{2}(\phi(x))=0$ for all $f \in C_{c}^{\infty}\left(W_{1}\right)$, which by Proposition 6.7 leads to a contradiction to the fact that $x \in M_{1}^{\text {int }}$. We conclude that $B \neq \emptyset$. We show that $B$ is closed in $M_{1}^{\text {int }}$ and thus $B=M_{1}^{\text {int }}$.

On $B$ we define

$$
J=\left(\phi^{*} R_{2}\right)^{-1} \circ R_{1}
$$

which is $C^{\infty}$ diffeomorphism $B \rightarrow J(B)$ by Lemma 6.10. We have for $x \in B$ and $f \in C_{c}^{\infty}\left(W_{2}\right)$ that $R_{1}(x)(f \circ \phi)=\left[\phi^{*} R_{2}\right](J(x))(f \circ \phi)$ and thus, by the definition (34),

$$
u_{f \circ \phi}^{1}(x)=u_{f}^{2}(J(x)), \quad x \in B .
$$

By (36), we directly also have that

$$
\left.J\right|_{W_{1}^{\text {int }}}=\phi: W_{1}^{\text {int }} \rightarrow W_{2}^{\text {int }}
$$

To show that $B$ is closed in $M_{1}^{\text {int }}$, we argue by contradiction and let $p_{k} \rightarrow x_{1} \in$ $\partial B \backslash B$, with $p_{k} \in B$. Then $x_{1} \in M_{1}^{\text {int }}$. By passing to a subsequence, we have $x_{2}:=$ $\lim _{k} J\left(p_{k}\right) \in M_{2}$. We have that $x_{2} \in M_{2}^{\text {int }}$; otherwise using (37) with $x=p_{k}$ and taking the limit as $k \rightarrow \infty$ would imply that $u_{f \circ \phi}^{1}\left(x_{1}\right)=u_{f}^{2}\left(x_{2}\right)=0$ for all $f \in C_{c}^{\infty}\left(W_{2}\right)$, i.e. $u_{h}^{1}\left(x_{1}\right)=0$ for all $h \in C_{c}^{\infty}\left(W_{1}\right)$, which would contradict Proposition 6.7(a). Let

$$
U=\left(u_{f_{1}}^{2}, u_{f_{2}}^{2}, \ldots, u_{f_{n}}^{2}\right)
$$

be solution coordinates as in Lemma 6.9 on a neighborhood $\Omega_{2} \subset M_{2}^{\text {int }}$ of the point $x_{2}$. Here $f_{j} \in C_{c}^{\infty}\left(W_{2}\right), j=1, \ldots, n$, and the limit $x_{2}$ is found by first passing to a subsequence.

We set

$$
V=\left(u_{f_{1} \circ \phi}^{1}, u_{f_{2} \circ \phi}^{1}, \ldots, u_{f_{n} \circ \phi}^{1}\right)
$$

We have $J=U^{-1} \circ V$ on $B$ near $x_{1}$ by (37). We have that $V$ is a solution coordinate system on a neighborhood $\Omega_{1} \subset M_{1}^{\text {int }}$ of $x_{1}$, and the "equation of injectivity"

$$
\left.d u_{f}^{1}\right|_{x_{1}} \cdot X=\left.d u_{f \circ \phi^{-1}}^{2}\right|_{x_{2}} \cdot D\left(U^{-1} \circ V\right) X, \quad f \in C_{c}^{\infty}\left(W_{1}\right),
$$

holds for $X \in T_{x_{1}} M_{1}$. These facts can be proved similarly following the argument in Theorem 3.3. We redefine the sets $\Omega_{1}$ and $\Omega_{2}$, if necessary, so that we have $V\left(\Omega_{1}\right)=$ $U\left(\Omega_{2}\right)$.

We next show that

$$
R_{1}(x)=\left(\phi^{*} R_{2}\right)\left(U^{-1} \circ V(x)\right), \quad x \in \Omega_{1} .
$$


This will imply

$$
R_{1}\left(B \cup \Omega_{1}\right) \subset\left(\phi^{*} R_{2}\right)\left(M_{2}^{\text {int }}\right),
$$

which is a contradiction and proves the theorem.

Let $f \in C_{c}^{\infty}\left(W_{1}\right)$. We claim that

$$
u_{f}^{1} \circ V^{-1}=u_{f \circ \phi^{-1}}^{2} \circ U^{-1} \text { on } V\left(\Omega_{1}\right) \subset \mathbb{R}^{n} .
$$

If $x_{1} \in W_{1}^{\text {int }}$ there is nothing to prove since then $x_{1} \in W_{1}^{\text {int }} \subset B$ and it is a contradiction to $x_{1} \in \partial B \backslash B$. Thus we may assume $x_{1} \in M_{1}^{\text {int }} \backslash \overline{\operatorname{supp}(f)}$. If $x_{2} \in W_{2}^{\text {int }}$, then by (38) and by the injectivity of $J$ and continuity of $J^{-1}$ we have that

$$
W_{1}^{\text {int }} \ni \phi^{-1}\left(x_{2}\right)=J^{-1}\left(x_{2}\right)=J^{-1} \lim _{k}\left(J\left(p_{k}\right)\right)=\lim _{k} J^{-1} J\left(p_{k}\right)=x_{1} .
$$

Thus we may also assume that $x_{2} \in M_{2}^{\text {int }} \backslash W_{2}^{\text {int }} \subset M_{2}^{\text {int }} \backslash \overline{\operatorname{supp}\left(f \circ \phi^{-1}\right)}$. It follows that there are small geodesic balls $\Omega_{1}^{\prime} \subset \Omega_{1}$ and $\Omega_{2}^{\prime} \subset \Omega_{2}$ of $x_{1}$ and $x_{2}$, respectively, such that $u_{f}^{1}$ and $u_{f \circ \phi^{-1}}^{2}$ are $C^{\omega}$ on $\Omega_{1}^{\prime}$ and $\Omega_{2}^{\prime}$, and that $V\left(\Omega_{1}^{\prime}\right) \subset U\left(\Omega_{2}^{\prime}\right)$. Consequently, the functions

$$
u_{f}^{1} \circ V^{-1}, u_{f \circ \phi^{-1}}^{2} \circ U^{-1}
$$

are real analytic on $V\left(\Omega_{1}^{\prime}\right)$.

The functions in (40) agree on $V\left(B \cap \Omega_{1}\right)$ by (37). Consequently, they agree on $V\left(\Omega_{1}^{\prime}\right)$ by real analyticity. The set $V\left(\Omega_{1}^{\prime}\right) \cap V\left(\Omega_{1} \backslash \bar{B}\right)$ is open and non-empty. Note that the functions in (40) are real analytic on $V\left(\Omega_{1} \backslash \bar{B}\right)$. This is because $\overline{\operatorname{supp}(f)} \subset$ $W_{1}^{\text {int }} \subset B$ and because $y \in \overline{\operatorname{supp}\left(f \circ \phi^{-1}\right)} \cap \Omega_{2}$ implies that

$$
\begin{aligned}
U(y) & \in U\left(W_{2}^{\text {int }} \cap \Omega_{2}\right) \subset U\left(J(B) \cap \Omega_{2}\right)=U\left(J\left(B \cap \Omega_{1}\right)\right) \\
& =U\left(\left(U^{-1} \circ V\right)\left(B \cap \Omega_{1}\right)\right)=V\left(B \cap \Omega_{1}\right) .
\end{aligned}
$$

By real analyticity the functions in (40) agree on $V\left(\Omega_{1} \backslash \bar{B}\right)$ and consequently on $V\left(\Omega_{1}\right)$. We have (39) since it is equivalent to

$$
u_{f}^{1} \circ V^{-1}=u_{f \circ \phi^{-1}}^{2} \circ U^{-1} \text { on } V\left(\Omega_{1}\right) \subset \mathbb{R}^{n} .
$$

Thus $B$ extends to a neighborhood of the point $x_{1} \in \partial B \backslash B$, which gives a contradiction. Thus $B$ is closed. Since $M_{1}^{\text {int }}$ is connected, we conclude that $B=M_{1}^{\text {int }}$.

By Lemma 6.10, $J$ is $C^{\infty}$ diffeomorphism $M_{1}^{\text {int }} \rightarrow J\left(M_{1}^{\text {int }}\right)$. Inverting the role of $M_{1}$ and $M_{2}$, we have $J\left(M_{1}^{\text {int }}\right)=M_{2}^{\text {int }}$. Since by Lemma 6.9 we may locally represent $J$ as $U^{-1} \circ V$ near any point, where $U$ and $V$ are $C^{\omega}$ solution coordinates, we have that $J$ and $J^{-1}$ are real analytic. If $u_{f}^{2}$ solves $L_{2} u_{f}^{2}=f$, where $f \in C_{c}^{\infty}\left(W_{2}\right)$ and $\left.u_{f}^{2}\right|_{\partial M}=0$, then (37) shows that $u_{f \circ \phi}^{1}=J^{*} u_{f}^{2}$. 


\subsection{Local determination of the coefficients}

We determine the coefficients of a quasilinear elliptic operator on open sets where the source-to-solution mapping of the operator is known. Precisely, we prove the following:

Proposition 6.11 Let $\left(M_{1}, g_{1}\right)$ and $\left(M_{2}, g_{2}\right)$ be compact connected Riemannian manifolds with mutual boundary and assume that $Q_{j}, j=1,2$, are quasilinear operators of the form (21) having coefficients $\mathcal{A}_{j}, \mathcal{B}_{j}$ satisfying (22)-(27).

Let $W_{j}, j=1,2$, be open subsets of $M_{j}$, and assume that there is a diffeomorphism $\phi: W_{1} \rightarrow W_{2}$ so that the source-to-solutions maps $S_{j}$ for $Q_{j}$ agree in the sense that

$$
\phi^{*} S_{2} f=S_{1} \phi^{*} f
$$

for all $f \in C_{c}^{\infty}\left(W_{2}\right)$ with $\|f\|_{C^{\alpha}\left(M_{2}\right)}$ sufficiently small.

Let $\widetilde{W} \subset \subset W_{1}^{\text {int }}$ be an open set. Then there is $\delta>0$ such that for all $x \in \widetilde{W}$ and $(c, \sigma) \in T_{x} \widetilde{W}$, with $|c|+|\sigma|_{g_{1}} \leq \delta$, we have

$$
\mathcal{A}_{1}(x, c, \sigma)=\phi^{*} \mathcal{A}_{2}(x, c, \sigma)=: \mathcal{A}(x, c, \sigma)
$$

and

$$
\mathcal{B}_{1}(x, c, \sigma)-\phi^{*} \mathcal{B}_{2}(x, c, \sigma)=\mathcal{A}^{a b}(x, c, \sigma)\left(\Gamma\left(g_{1}\right)_{a b}^{k}-\Gamma\left(\phi^{*} g_{2}\right)_{a b}^{k}\right) \sigma_{k}
$$

To prove the proposition, we begin with the following observation.

Lemma 6.12 Assume the conditions and notation in Proposition 6.11. Let $x \in W_{1}^{\text {int }}$ and let $U_{2}$ be coordinates on a neighborhood $\Omega_{2} \subset \subset W_{2}$ of $\phi(x) \in W_{2}^{\text {int }}$. Let $U_{1}=\phi^{*} U_{2}$ be coordinates on a neighborhood $\phi^{-1}\left(\Omega_{2}\right) \subset \subset W_{1}$ of the point $x$.

Denote $\Omega=U_{2}\left(\Omega_{2}\right) \subset \mathbb{R}^{n}$. There is $\delta^{\prime}>0$ such that for all $v \in C_{c}^{\infty}(\Omega)$ with $\|v\|_{C^{2, \alpha}(\Omega)} \leq \delta^{\prime}$ we have

$$
\tilde{Q}_{1} v=\tilde{Q}_{2} v \text { on } \Omega
$$

Here $\tilde{Q}_{j}$ are the coordinate representations of $Q_{j}$ in coordinates $U_{j}, j=1,2$.

Proof Let $x \in W_{1}^{\text {int }}$ and let $U_{1}$ and $U_{2}$ be coordinate systems as in the statement of the lemma. Let $\varepsilon_{j}, \delta_{j}>0, j=1,2$, be as in the Definitions (28)-(30) of the source-tosolution mapping of $Q_{j}$. Set $\varepsilon=\min \left(\varepsilon_{1}, \varepsilon_{2}\right)$. Let $\delta^{\prime}>0$ be such that if $v \in C_{c}^{\infty}(\Omega)$ satisfy $\|v\|_{C^{2, \alpha}(\Omega)} \leq \delta^{\prime}$, then

$$
\min \left(\left\|Q_{2}\left(v \circ U_{2}\right)\right\|_{C^{\alpha}\left(W_{2}\right)},\left\|\phi^{*}\left(Q_{2}\left(v \circ U_{2}\right)\right)\right\|_{C^{\alpha}\left(W_{1}\right)}\right) \leq \varepsilon
$$

and

$$
\left\|v \circ U_{2}\right\|_{C^{2, \alpha}\left(W_{2}\right)} \leq \delta_{2}
$$


Such a $\delta^{\prime}$ can be found since $\mathcal{B}_{2}(x, 0,0)=0$ by Assumption (25), since $Q_{2}$ : $C^{2, \alpha}\left(W_{2}\right) \rightarrow C^{\alpha}\left(W_{2}\right)$ is continuous and since composing a compactly supported function with $C^{\infty}$ diffeomorphism is continuous on Hölder spaces.

Let $v \in C_{c}^{\infty}(\Omega)$ with $\|v\|_{C^{2, \alpha}(\Omega)} \leq \delta^{\prime}$. Then the problem $Q_{2} u_{F}^{2}=F$ in $M_{2}$ with $\left.u_{F}^{2}\right|_{\partial M}=0$ where

$$
F:=Q_{2}\left(v \circ U_{2}\right) \in C_{c}^{\infty}\left(W_{2}\right)
$$

has a unique solution $u_{F}^{2}$ with $\left\|u_{F}^{2}\right\|_{C^{2, \alpha}\left(M_{2}\right)} \leq \delta_{2}$ by Proposition 6.1. Let us extend $v \circ U_{2}$ by zero onto $M_{2}$. By the condition $\mathcal{B}_{2}(x, 0,0)=0$ and we have

$$
Q_{2}\left(v \circ U_{2}\right)=F \text { on } M_{2} \text { and } v \circ U_{2}=0 \text { on } \partial M \text {. }
$$

Since $\left\|v \circ U_{2}\right\|_{C^{2, \alpha}\left(M_{2}\right)} \leq \delta_{2}$, by uniqueness we have that

$$
u_{F}^{2}=v \circ U_{2}
$$

By the definition of $\delta^{\prime}$ we have that $Q_{1} u_{F}^{1}=\phi^{*} F$ with $\left.u_{F}^{1}\right|_{\partial M}=0$ has a unique solution with $\left\|u_{F}^{1}\right\|_{C^{2, \alpha}\left(M_{1}\right)} \leq \delta_{1}$. Since the source-to-solutions mappings agree, we have

$$
\left.u_{F}^{1}\right|_{W_{1}}=S_{1} \phi^{*} F=\phi^{*} S_{2} F=\phi^{*}\left(\left.u_{F}^{2}\right|_{W_{2}}\right) .
$$

By using $\left.\phi\right|_{\Omega_{1}}=U_{2}^{-1} \circ U_{1}$ it follows that

$$
u_{F}^{1} \circ U_{1}^{-1}=u_{F}^{2} \circ U_{2}^{-1} \text { on } \Omega .
$$

We also have on $W_{2}$ that

$$
Q_{2} u_{F}^{2}=F=F \circ \phi \circ \phi^{-1}=\left(Q_{1} u_{F}^{1}\right) \circ \phi^{-1} .
$$

Using the coordinate invariance of $Q_{j}$, we have by (43)-(45) that

$$
\begin{aligned}
\tilde{Q}_{2} v & =\tilde{Q}_{2}\left(u_{F}^{2} \circ U_{2}^{-1}\right)=\left(Q_{2} u_{F}^{2}\right) \circ U_{2}^{-1}=\left(Q_{1} u_{F}^{1}\right) \circ\left(U_{2} \circ \phi\right)^{-1} \\
& =\left(Q_{1} u_{F}^{1}\right) \circ U_{1}^{-1}=\tilde{Q}_{1}\left(u_{F}^{1} \circ U_{1}^{-1}\right)=\tilde{Q}_{1}\left(u_{F}^{2} \circ U_{2}^{-1}\right)=\tilde{Q}_{1} v .
\end{aligned}
$$

The lemma tells us that we can use any test function $v$ with small enough $C^{2, \alpha}$ norm to solve for the coefficients of $\tilde{Q}_{j}$ from the equation $\tilde{Q}_{1} v=\tilde{Q}_{2} v$ in the coordinates $U_{1}$ and $U_{2}$. The local determination result of Proposition 6.11 is a consequence of this observation. Its proof is similar to that of Proposition 4.1 where we used harmonic functions to solve for (a multiple of) the metric in the Calderón problem. 
Proof of Proposition 6.11 Let $x_{0} \in W_{1}^{\text {int }}$ and let $U_{2}$ be coordinates on a neighborhood $\Omega_{2} \subset \subset W_{2}^{\text {int }}$ of $\phi\left(x_{0}\right) \in W_{2}^{\text {int }}$ and let $U_{1}=\phi^{*} U_{2}$ be coordinates on a neighborhood $\Omega_{1}=\phi^{-1}\left(\Omega_{2}\right)$ of $x_{0}$. Denote $\Omega=U_{2}\left(\Omega_{2}\right)=U_{1}\left(\Omega_{1}\right)$. By the Lemma 6.12 above, we have that there is $\delta^{\prime}>0$ such that for all $v \in C_{c}^{\infty}(\Omega)$ with $\|v\|_{C^{2, \alpha}(\Omega)} \leq \delta^{\prime}$ we have

$$
\tilde{Q}_{1} v=\tilde{Q}_{2} v
$$

Here $\tilde{Q}_{j}$ are the coordinate representations of $Q_{j}$ in coordinates $U_{j}, j=1,2$.

We construct the test functions we use. We may assume that $U_{1}\left(x_{0}\right)=U_{2}\left(\phi\left(x_{0}\right)\right)=$ 0 . Let $r>0$ so that $B(0, r) \subset \subset \Omega \subset \mathbb{R}^{n}$, and let $\chi: \Omega \rightarrow \mathbb{R}$ be a cutoff function, which is 1 on $\bar{B}(0, r)$ and vanishes outside $\bar{B}\left(0, r^{\prime}\right) \subset \subset \Omega$ for some $r^{\prime}>r$. Define $\widehat{\Omega}=U_{2}^{-1}(B(0, r))$. If $y \in \widehat{\Omega}$ and $(c, \sigma) \in \mathbb{R} \times T_{y}^{*} \widehat{\Omega}$, define a function $v=v_{(y, c, \sigma, A)} \in$ $C_{c}^{\infty}(\Omega)$ in the coordinates $U_{2}$ as

$$
v(x)=\chi(x)\left(c+\sigma \cdot(x-y)+\frac{1}{2} A(x-y) \cdot(x-y)\right),
$$

where $A$ is a symmetric $n \times n$-matrix. Then we have

$$
v(y)=c, d v(y)=\sigma \text { and } \partial_{a b} v(y)=A_{a b} .
$$

There is $\delta>0$ such that for all $y \in \widehat{\Omega}$ and $(c, \sigma) \in \mathbb{R} \times T_{y}^{*} \widehat{\Omega}$ with $|c|+|\sigma|_{g_{2}}+\|A\| \leq$ $2 \delta$, we have

$$
\left\|v_{(y, c, \sigma, A)}\right\|_{C^{2, \alpha}(\Omega)}<\delta^{\prime}
$$

Here $\|A\|$ is the Hilbert-Schmidt norm of matrices and $\delta$ is independent of $y \in \widehat{\Omega}$.

Let $y \in \widehat{\Omega}$ and let $(c, \sigma) \in \mathbb{R} \times T_{y}^{*} \widehat{\Omega}$ with $|c|+|\sigma|_{g_{2}} \leq \delta$. Let $A$ be a matrix with $\|A\| \leq \delta$ and let $v=v_{(y, c, \sigma, A)}$ be the function defined in (47). Since $\|v\|_{C^{2, \alpha}(\Omega)}<\delta^{\prime}$ by (48), Eq. 46 implies that

$$
\begin{aligned}
& \mathcal{A}_{1}(x, v(x), d v(x))^{a b}\left(\partial_{a b} v(x)-\Gamma_{1, a b}^{k}(x) \partial_{k} v(x)\right)+\mathcal{B}_{1}(x, v(x), d v(x)) \\
& \quad=\mathcal{A}_{2}(x, v(x), d v(x))^{a b}\left(\partial_{a b} v(x)-\Gamma_{2, a b}^{k}(x) \partial_{k} v(x)\right)+\mathcal{B}_{2}(x, v(x), d v(x)),
\end{aligned}
$$

for $x \in \Omega$. Here $\Gamma_{j, a b}^{k}$ are the Christoffel symbols of $g_{j}$, and $\mathcal{A}_{j}$ and $\mathcal{B}_{j}$ are understood as their coordinate representations in coordinates $U_{j}$.

Let us first choose as the matrix $A$ a one with $\|A\| \leq \delta$ and that satisfies have

$$
A \perp \mathcal{A}_{1}(y, v(y), d v(y))-\mathcal{A}_{2}(y, v(y), d v(y)) .
$$

Here $\perp$ is defined with respect to the Hilbert-Schmidt inner product of matrices. We have $\|v\|_{C^{2, \alpha}(\Omega)} \leq 2 \delta$ and thus Eq. 49 holds. It follows that

$$
\mathcal{B}_{1}(y, c, \sigma)-\mathcal{B}_{2}(y, c, \sigma)=\left(\mathcal{A}_{1}^{a b}(y, c, \sigma) \Gamma_{1, a b}^{k}(y)-\mathcal{A}_{2}^{a b}(y, c, \sigma) \Gamma_{2, a b}^{k}(y)\right) \sigma_{k} .
$$


We next choose the matrix $A$ as

$$
A_{a b}=\rho\left(\mathcal{A}_{1}^{a b}(y, c, \sigma)-\mathcal{A}_{2}^{a b}(y, c, \sigma)\right)
$$

where $\rho>0$ is a number small enough so that $\|A\| \leq \delta$. It follows from (49) and by using (50) that

$$
\rho\left\|\mathcal{A}_{1}(y, c, \sigma)-\mathcal{A}_{2}(y, c, \sigma)\right\|^{2}=0
$$

where $\|\cdot\|$ is the Hilbert-Schmidt norm of matrices. Thus

$$
\mathcal{A}_{1}^{a b}(y, c, \sigma)=\mathcal{A}_{2}^{a b}(y, c, \sigma),
$$

which combined with (50), yields

$$
\mathcal{B}_{1}(y, c, \sigma)-\mathcal{B}_{2}(y, c, \sigma)=\mathcal{A}_{1}^{a b}(y, c, \sigma)\left(\Gamma_{1, a b}^{k}(y)-\Gamma_{2, a b}^{k}(y)\right) \sigma_{k} .
$$

Equations (51) and (52) hold for all $(y, c, \sigma) \in \widehat{\Omega} \times \mathbb{R} \otimes T^{*} \widehat{\Omega}$ with $|c|+|\sigma|_{g_{2}} \leq \delta$. Recall that (51) and (52) are equations for the coordinate representations of $\mathcal{A}_{1}, \mathcal{B}_{1}$ and $\mathcal{A}_{2}, \mathcal{B}_{2}$ in coordinates $U_{1}$ and $U_{2}$ respectively, where $U_{1}=\phi^{*} U_{2}$. Thus we have, by redefining $\delta$ if necessary, that

$$
\begin{aligned}
& \mathcal{A}_{1}(x, c, \sigma)=\phi^{*} \mathcal{A}_{2}(x, c, \sigma)=: \mathcal{A}(x, c, \sigma) \\
& \mathcal{B}_{1}(x, c, \sigma)-\phi^{*} \mathcal{B}_{2}(x, c, \sigma)=\mathcal{A}^{a b}(x, c, \sigma)\left(\Gamma\left(g_{1}\right)_{a b}^{k}-\Gamma\left(\phi^{*} g_{2}\right)_{a b}^{k}\right) \sigma_{k},
\end{aligned}
$$

for $(x, c, \sigma) \in\left(\phi^{-1} \widehat{\Omega}\right) \times \mathbb{R} \otimes T^{*}\left(\phi^{-1} \widehat{\Omega}\right)$ with $|c|+|\sigma|_{g_{1}} \leq \delta$. Let $\widetilde{W}$ be an open set compactly contained in $W_{1}^{\text {int }}$. Since we may carry out the argument above on a neighborhood of any point $x_{0} \in W_{1}^{\text {int }}$, we have the claim by compactness for some $\delta>0$.

\subsection{Global determination of the coefficients}

We prove the main theorem of this section.

Proof of Theorem 6.2 By Theorem 6.5 we know that there is a real analytic diffeomorphism $J: M_{1}^{\text {int }} \rightarrow M_{2}^{\text {int }}$ that satisfies $\left.J\right|_{W_{1}^{\text {int }}}=\phi: W_{1}^{\text {int }} \rightarrow W_{2}^{\text {int }}$. It follows that $J^{*} \mathcal{A}_{2}$ and $J^{*} \mathcal{B}_{2}$, which are given by

$$
\left(J^{*} \mathcal{A}_{2}\right)(x, c, \sigma)=\left.\left.\left((D J)^{-1}\right)^{T}\right|_{J(x)} \mathcal{A}_{2}\left(J(x), c, J^{-1 *} \sigma\right)(D J)^{-1}\right|_{J(x)}
$$

and

$$
\left(J^{*} \mathcal{B}_{2}\right)(x, c, \sigma)=\mathcal{B}_{2}\left(J(x), c, J^{-1 *} \sigma\right),
$$


are real analytic for all $x \in M_{1}^{\text {int }}$ and $(c, \sigma) \in \mathbb{R} \times T_{x}^{*} M_{1}^{\text {int }}$. By Proposition 6.11 we have that there is a non-empty open set $\widetilde{W} \subset W_{1}^{\text {int }}{ }^{x}$ and $\delta>0$ such that for all $(c, \sigma) \in \mathbb{R} \times T_{x}^{*} \widetilde{W}$ with $|c|+|\sigma|_{g_{1}} \leq \delta$, we have

$$
\begin{aligned}
& \mathcal{A}_{1}(x, c, \sigma)=J^{*} \mathcal{A}_{2}(x, c, \sigma) \text { and } \\
& \mathcal{B}_{1}(x, c, \sigma)-J^{*} \mathcal{B}_{2}(x, c, \sigma)=\mathcal{A}^{a b}(x, c, \sigma)\left(\Gamma\left(g_{1}\right)_{a b}^{k}(x)-\Gamma\left(J^{*} g_{2}\right)_{a b}^{k}(x)\right) \sigma_{k},
\end{aligned}
$$

where $\mathcal{A}=\mathcal{A}_{1}=J^{*} \mathcal{A}_{2}$. By real analyticity, we have (54) for all $x \in M_{1}^{\text {int }}$ and $(c, \sigma) \in \mathbb{R} \times T_{x}^{*} M_{1}^{\text {int }}$.

Proof of Corollary 6.3 (1) Let $(x, c, \sigma) \in M_{1}^{\text {int }} \times \mathbb{R} \otimes T^{*} M_{1}^{\text {int }}$. Assume that $\mathcal{A}_{1}(x, c, \sigma)$ is $s$-homogeneous in the $\sigma$-variable and that $\mathcal{B}_{1}$ and $\mathcal{B}_{2}$ are $s^{\prime}$-homogeneous with $s^{\prime} \neq s+1$. It follows from (53) that $J^{*} \mathcal{B}_{2}$

is $s^{\prime}$-homogeneous in the $\sigma$-variable. By Theorem 6.2 we know that $J^{*} \mathcal{A}_{2}=\mathcal{A}_{1}$ is $s+1$ homogeneous in the $\sigma$-variable. Thus

$$
\Gamma(x, c, \sigma):=\mathcal{A}^{a b}(x, c, \sigma)\left(\Gamma\left(g_{1}\right)_{a b}^{k}(x)-\Gamma\left(J^{*} g_{2}\right)_{a b}^{k}(x)\right) \sigma_{k},
$$

where $\mathcal{A}=\mathcal{A}_{1}=J^{*} \mathcal{A}_{2}$, is $(s+1)$-homogeneous in the $\sigma$-variable. Since

$$
\mathcal{B}(x, c, \sigma):=\mathcal{B}_{1}(x, c, \sigma)-J^{*} \mathcal{B}_{2}(x, c, \sigma)
$$

is $s^{\prime}$-homogeneous, $s^{\prime} \neq s+1$, in the $\sigma$-variable, we must have

$$
\mathcal{B}_{1}-J^{*} \mathcal{B}_{2}=0 \text { and } \Gamma(x, c, \sigma)=0
$$

by Theorem 6.2.

(2) Assume that $\phi$ is an isometry. Since $\left.J\right|_{W_{1}^{\text {int }}}=\left.\phi\right|_{W_{1}^{\text {int }}}$, we have $\Gamma\left(g_{1}\right)_{a b}^{k}=$ $\Gamma\left(\phi^{*} g_{2}\right)_{a b}^{k}=\Gamma\left(J^{*} g_{2}\right)_{a b}^{k}$ on $W_{1}^{\text {int }}$. Since $\Gamma\left(g_{1}\right)_{a b}^{k}-\Gamma\left(J^{*} g_{2}\right)_{a b}^{k}$ is a real analytic tensor field, which vanishes on $W_{1}^{\text {int }}$, it vanishes on $M_{1}^{\text {int }}$. Thus $\Gamma(x, c, \sigma)=0=\mathcal{B}(x, c, \sigma)$ for all $(x, c, \sigma) \in M_{1}^{\text {int }} \times \mathbb{R} \otimes T^{*} M_{1}^{\text {int }}$.

Acknowledgements Open access funding provided by University of Jyväskylä (JYU). M.L., T.L. and M.S. were supported by the Academy of Finland (Centre of Excellence in Inverse Modelling and Imaging, grant numbers 284715 and 309963). M.S. was also partly supported by the European Research Council under FP7/2007-2013 (ERC StG 307023) and Horizon 2020 (ERC CoG 770924). We would like to thank an anonymous referee for valuable comments.

Open Access This article is distributed under the terms of the Creative Commons Attribution 4.0 International License (http://creativecommons.org/licenses/by/4.0/), which permits unrestricted use, distribution, and reproduction in any medium, provided you give appropriate credit to the original author(s) and the source, provide a link to the Creative Commons license, and indicate if changes were made. 


\section{Appendix A: Runge approximation results}

Here we prove the Runge approximation results that are used repeatedly in this paper. In this section, which is independent of the other sections, we will assume that $(M, g)$ is a compact connected oriented Riemannian manifold with boundary, and $\operatorname{dim}(M) \geq 2$. We will mostly use the following easy consequence of Runge approximation.

Proposition A.1 Let $\Gamma$ be a nonempty open subset of $\partial$, and denote by $u_{f}$ the solution of $\Delta_{g} u=0$ in $M$ with $\left.u\right|_{\partial M}=f$.

(a) If $x \in M^{\text {int }} \cup \Gamma, y \in M$ and $x \neq y$, there is $f \in C_{c}^{\infty}(\Gamma)$ such that

$$
u_{f}(x) \neq u_{f}(y)
$$

(b) If $x \in M^{\mathrm{int}} \cup \Gamma$ and $v \in T_{x}^{*} M$, there is $f \in C_{c}^{\infty}$ (Г) such that

$$
d u_{f}(x)=v .
$$

The main Runge approximation result is the following.

Proposition A.2 Let $s \geq 1$, let $\Gamma$ be a nonempty open subset of $\partial M$, and let $U \subset \subset M^{\text {int }}$ be a domain with $C^{\infty}$ boundary such that $M \backslash \bar{U}$ is connected. Let also L be a second order uniformly elliptic differential operator on $M$, and let $\mathcal{P}$ be the Poisson operator for L. Then the set

$$
\mathcal{R}=\left\{\left.\mathcal{P} f\right|_{U} ; f \in C_{c}^{\infty}(\Gamma)\right\}
$$

is dense in the space $\mathcal{S}=\left\{u \in H^{s}(U): L u=0\right.$ in $\left.U\right\}$ with respect to the $H^{s}(U)$ norm.

The proof is a standard Runge approximation argument, which boils down to the solvability of the adjoint equation in negative order Sobolev spaces and the unique continuation property. It will be convenient to embed $(M, g)$ in a closed manifold $(N, g)$ and to extend the operator $L$ in $M$ as a second order uniformly elliptic operator $A$ in $N$. In this way we avoid having to consider boundary values of solutions in negative order Sobolev spaces. The required solvability result will follow from the next lemma, where $A^{*}$ is the formal $L^{2}$-adjoint.

Lemma A.3 Let $(N, g)$ be a closed manifold, and let $A$ be an elliptic second order differential operator on $N$ with kernel $\mathcal{N}=\left\{v \in C^{\infty}(N) ; A v=0\right\}$.

If $s \in \mathbb{R}$, then for any $F \in H^{-s}(N)$ satisfying $(F, v)_{N}=0$ for all $v \in \mathcal{N}$ there is a unique solution $u \in H^{-s+2}(N)$ with minimal $H^{-s+2}(N)$ norm of

$$
A^{*} u=F \text { in } N
$$

One also has $\|u\|_{H^{-s+2}(N)} \leq C\|F\|_{H^{-s}(N)}$ with $C$ independent of $F$. Morever, if $\left.F\right|_{U}=0$ for some open set $U \subset N$, then for any $m \geq 0$ and any $V \subset \subset U$ one has

$$
\|u\|_{H^{m}(V)} \leq C_{m, V}\|F\|_{H^{-s}(N)} .
$$


Proof Let $r \in \mathbb{R}$, and consider the map

$$
T_{r}: H^{r+2}(N) \rightarrow H^{r}(N), \quad T_{r} u=A^{*} u .
$$

By [23, Theorem 19.2.1] this map is a Fredholm operator with finite dimensional kernel $\mathcal{N}^{*}:=\left\{v \in C^{\infty}(N) ; A^{*} v=0\right\}$, and the range of $T_{r}$ is given by

$$
\operatorname{Ran}\left(T_{r}\right)=\left\{w \in H^{r}(N) ;(w, v)_{N}=0 \text { for } v \in \mathcal{N}\right\}
$$

In particular, the kernel and cokernel are independent of $r$. Writing $E$ for the orthocomplement of $\mathcal{N}^{*}$ in $H^{-s+2}(N)$ we get that the operator $T: E \rightarrow A^{*}\left(H^{-s+2}(N)\right), T u=$ $A^{*} u$ is continuous and bijective, hence invertible by the open mapping theorem, and $u=T^{-1} F$ is the unique minimal norm solution of $A^{*} u=F$. We will also write $u=\left(A^{*}\right)^{-1} F$. This proves the first statement.

Assume now that $\left.F\right|_{U} \equiv 0$, let $V \subset \subset U$ and let $m \geq 0$. Let $\chi, \chi_{1} \in C_{c}^{\infty}(U)$ satisfy $\chi=1$ near $\bar{V}$ and $\chi_{1}=1$ near $\operatorname{supp}(\chi)$. Let also $Q \in \Psi^{-2}(N)$ be a parametrix for $A^{*}$, so that

$$
Q A^{*}=\mathrm{Id}+R
$$

where $R \in \Psi^{-\infty}(N)$. Then the minimal norm solution $u=\left(A^{*}\right)^{-1} F$ of $A^{*} u=F$ in $N$ satisfies $u=Q F-R u=Q F-R\left(A^{*}\right)^{-1} F$. Consequently

$$
\begin{aligned}
\|u\|_{H^{m}(V)} & \leq\|\chi u\|_{H^{m}(N)} \leq\|\chi Q F\|_{H^{m}(N)}+\left\|\chi R\left(A^{*}\right)^{-1} F\right\|_{H^{m}(N)} \\
& \leq\left\|\chi Q\left(1-\chi_{1}\right) F\right\|_{H^{m}(N)}+\left\|\chi R\left(A^{*}\right)^{-1} F\right\|_{H^{m}(N)}
\end{aligned}
$$

using that $\left.F\right|_{U} \equiv 0$. Using the pseudolocal property of $Q$ and the fact that $R$ is smoothing, we get the estimate

$$
\|u\|_{H^{m}(V)} \leq C\|F\|_{H^{-s}(N)} .
$$

We will also need the following simple lemma.

Lemma A.4 Let $(N, g)$ be a closed manifold, let A be a second order uniformly elliptic differential operator on $N$, let $\mathcal{N}$ be the kernel of $A$, and let $\left\{\psi_{1}, \ldots, \psi_{m}\right\}$ be an $L^{2}(N)$-orthonormal basis of $\mathcal{N}$. Let also $V \subset N$ be any nonempty open set. There exist $\eta_{1}, \ldots, \eta_{m} \in C_{c}^{\infty}(V)$ such that

$$
\left(\eta_{j}, \psi_{k}\right)_{N}=\delta_{j k}, \quad 1 \leq j, k \leq m
$$

Proof Consider the map

$$
T: L^{2}(V) \rightarrow \mathbb{R}^{m}, \quad T \eta=\left(\left(\eta, \psi_{1}\right)_{V}, \ldots,\left(\eta, \psi_{m}\right)_{V}\right)
$$


Then $T\left(\left.a_{1} \psi_{1}\right|_{V}+\cdots+\left.a_{m} \psi_{m}\right|_{V}\right)=S \mathbf{a}$, where $S=\left(\left(\psi_{j}, \psi_{k}\right)_{V}\right)_{j, k=1}^{m}$. Now if $S \mathbf{a}=0$, then in particular $S \mathbf{a} \cdot \mathbf{a}=\left\|a_{1} \psi_{1}+\cdots+a_{m} \psi_{m}\right\|_{L^{2}(V)}^{2}=0$ and thus $\left.a_{1} \psi_{1}\right|_{V}+\cdots+\left.a_{m} \psi_{m}\right|_{V}=0$. It follows that $a_{1} \psi_{1}+\cdots+a_{m} \psi_{m}=0$ in $N$ by elliptic unique continuation, showing that $\mathbf{a}=0$ and that $S$ is invertible.

Since the matrix $S$ is invertible, $T$ is surjective. Finally, since $C_{c}^{\infty}(V)$ is dense in $L^{2}(V)$ we have that $T\left(C_{c}^{\infty}(V)\right)$ is a dense subspace of $\mathbb{R}^{m}$. Since $\mathbb{R}^{m}$ is finite dimensional, it follows that $T\left(C_{c}^{\infty}(V)\right)=\mathbb{R}^{m}$. We can thus choose $\eta_{j} \in C_{c}^{\infty}(V)$ with $T \eta_{j}=e_{j}$ for $1 \leq j \leq m$.

Proof of Proposition A.2 Let $F \in\left(H^{s}(U)\right)^{*}$ satisfy $F\left(\left.\mathcal{P} f\right|_{U}\right)=0$ for all $f \in C_{c}^{\infty}(\Gamma)$. By the Hahn-Banach theorem, it is enough to show that $F(u)=0$ for all $u \in \mathcal{S}$.

Let $(M, g) \subset \subset\left(M_{1}, g\right) \subset \subset(N, g)$, where $M_{1}$ is a compact manifold with boundary and $N$ is a closed connected manifold. We extend the coefficients of $L$ to $N$ without destroying the ellipticity to define an elliptic second order operator $A$ in $N$ (thus $\left.A w\right|_{M}=L\left(\left.w\right|_{M}\right)$ for $\left.w \in C^{\infty}(N)\right)$. Also, define $\hat{F} \in H^{-s}(N)$ by

$$
\hat{F}(w)=F\left(\left.w\right|_{U}\right), \quad w \in H^{s}(N) .
$$

It follows that $\operatorname{supp}(\hat{F}) \subset \bar{U}$. If $\hat{F}$ is not orthogonal to the kernel $\mathcal{N}$ of $A$, we need to modify it outside $M_{1}$ as follows. Let $V$ be an open subset of $N \backslash M_{1}$, let $\left\{\psi_{1}, \ldots, \psi_{m}\right\}$ and $\left\{\eta_{1}, \ldots, \eta_{m}\right\}$ be as in Lemma A.4, and define

$$
\eta=-\sum_{j=1}^{m} \hat{F}\left(\psi_{j}\right) \eta_{j}
$$

We define $\tilde{F} \in H^{-s}(N)$ with $\operatorname{supp}(\tilde{F}) \subset \bar{U} \cup \bar{V}$ by

$$
\tilde{F}=\hat{F}+\eta \text {. }
$$

Lemma A.4 ensures that $\tilde{F}\left(\psi_{k}\right)=0$ for $1 \leq k \leq m$.

Now, by Lemma A.3 there is a unique minimal norm solution $v \in H^{-s+2}(N)$ of

$$
A^{*} v=\tilde{F} \text { in } N \text {. }
$$

It will be convenient to choose smooth approximations $\tilde{F}_{j}=\hat{F}_{j}+\eta_{j}$, where $\hat{F}_{j} \in$ $C_{c}^{\infty}\left(M^{\text {int }}\right)$ has support near $\bar{U}$ and $\hat{F}_{j} \rightarrow \hat{F}$ in $H^{-s}(N)$ and $\eta_{j} \in C_{c}^{\infty}(V)$ is defined as in (55) but with $\hat{F}$ replaced by $\hat{F}_{j}$. Then $\tilde{F}_{j} \rightarrow \tilde{F}$ in $H^{-s}(N)$ as $j \rightarrow \infty$. We let $v_{j} \in C^{\infty}(N)$ be the minimal norm solution of

$$
A^{*} v_{j}=\tilde{F}_{j} \text { in } N
$$

In particular, one has $A^{*} v=0$ near $\partial M$, so $v$ is $C^{\infty}$ near $\partial M$ by elliptic regularity. We will next solve

$$
L^{*} w_{j}=0 \text { in } M,\left.\quad w_{j}\right|_{\partial M}=\left.v_{j}\right|_{\partial M}
$$




$$
L^{*} w=0 \text { in } M,\left.\quad w\right|_{\partial M}=\left.v\right|_{\partial M} .
$$

Define $\varphi_{j}=\left.v_{j}\right|_{M}-w_{j}$ and $\varphi=\left.v\right|_{M}-w$. It follows that $\varphi_{j} \in C^{\infty}(M)$ and $\varphi \in H^{-s+2}(M)$ solve

$$
\begin{aligned}
& L^{*} \varphi_{j}=\tilde{F}_{j} \text { in } M^{\text {int }},\left.\quad \varphi_{j}\right|_{\partial M}=0, \\
& L^{*} \varphi=\tilde{F} \text { in } M^{\text {int }},\left.\quad \varphi\right|_{\partial M}=0 .
\end{aligned}
$$

Let $E$ be a bounded extension operator $H^{s}(M) \rightarrow H^{S}(N)$ that satisfies $\operatorname{supp}(E w) \subset M_{1}^{\text {int }}$ for all $w \in H^{s}(M)$. Since $F\left(\left.\mathcal{P} f\right|_{U}\right)=0$ for all $f \in C_{c}^{\infty}(\Gamma)$, we have

$$
\begin{aligned}
0 & =F\left(\left.\mathcal{P} f\right|_{U}\right)=\tilde{F}(E(\mathcal{P} f))=\lim \tilde{F}_{j}(E(\mathcal{P} f)) \\
& =\lim \left(A^{*} v_{j}, E(\mathcal{P} f)\right)_{N} .
\end{aligned}
$$

In the last expression, $E(\mathcal{P} f)$ is supported in $M_{1}^{\text {int }}$ and $\left.A^{*} v_{j}\right|_{M_{1}}$ vanishes outside a neighborhood of $\bar{U}$. Thus this expression can be understood as an integral over $M$. Integrating by parts, we get

$$
\begin{aligned}
0 & =\lim \left(A^{*} v_{j}, \mathcal{P} f\right)_{M}=\lim \left(A^{*} \varphi_{j}, \mathcal{P} f\right)_{M} \\
& =\lim \left(\partial_{v}^{A^{*}} \varphi_{j}, f\right)_{\partial M .}
\end{aligned}
$$

Here $\partial_{v}^{A^{*}}$ is the normal derivative associated with $A^{*}$, and we used that $\left.\varphi_{j}\right|_{\partial M}=0$. Now, if $W$ is a small neighborhood of $\partial M$, the higher regularity estimate in Lemma A.3 implies that $v_{j} \rightarrow v$ in $H^{m}(W)$ for any $m \geq 0$. Thus one has $\partial_{v}^{A^{*}} \varphi_{j} \rightarrow \partial_{v}^{A^{*}} \varphi$ in $H^{m}(\partial M)$ for any $m \geq 0$, and consequently

$$
0=\left(\partial_{v}^{A^{*}} \varphi, f\right)_{\partial M}
$$

Since the previous result holds for all $f \in C_{c}^{\infty}(\Gamma)$, we see that $\varphi$ solves

$$
L^{*} \varphi=0 \text { in } M^{\text {int }} \backslash \bar{U},\left.\quad \varphi\right|_{\partial M}=0,\left.\quad \partial_{v}^{A^{*}} \varphi\right|_{\Gamma}=0
$$

Also, $\varphi$ is smooth in $M \backslash \bar{U}$. The unique continuation principle implies that $\left.\varphi\right|_{M \backslash \bar{U}}=0$. Thus $\varphi$ may be identified with an element of $H_{\bar{U}}^{-s+2}(N)$ (the distributions in $H^{-s+2}(N)$ supported in $\bar{U})$, and it follows that there exist $\psi_{j} \in C_{c}^{\infty}(U)$ with $\psi_{j} \rightarrow \varphi$ in $H^{-s+2}(N)$ (see e.g. [32, Theorem 3.29]).

Finally, let $u \in \mathcal{S}$, and let $E$ be a bounded extension operator from $H^{s}(U)$ to $H^{s}(N)$ so that $\operatorname{supp}(E w) \subset M^{\text {int }}$ for any $w \in H^{s}(U)$. Then, since $\varphi \in H_{\bar{U}}^{-s+2}(N)$,

$$
\begin{aligned}
F(u) & =\tilde{F}(E u)=\left(A^{*} \varphi, E u\right)_{N} \\
& =\lim \left(A^{*} \psi_{j}, E u\right)_{N}=\lim \left(A^{*} \psi_{j}, u\right)_{U} .
\end{aligned}
$$


Using that $\psi_{j} \in C_{c}^{\infty}(U)$ and $L u=0$ in $U$, we may integrate by parts in $U$ and obtain that $F(u)=0$ for all $u \in \mathcal{S}$. This concludes the proof.

As a consequence of the Runge approximation property we can find global harmonic functions with prescribed 2-jet at a given point, up to the natural restrictions given by the equation $\Delta_{g} u=0$. The existence of a local harmonic function with this property is found e.g. in [1, Lemma A.1.1]), but we give the details for completeness.

Proposition A.5 Let $\Gamma$ be a nonempty open subset of $\partial M$. Let $p \in M^{\text {int }} \cup \Gamma$, let $a_{0} \in \mathbb{R}$, let $\xi_{0} \in T_{p}^{*} M$, and let $H_{0}$ be a symmetric 2-tensor at $p$ satisfying $\operatorname{Tr}_{g}\left(H_{0}\right)=0$. There exists $f \in C_{c}^{\infty}(\Gamma)$ such that the solution of

$$
-\Delta_{g} u=0 \text { in } M,\left.\quad u\right|_{\partial M}=f
$$

satisfies $u(p)=a_{0}, d u(p)=\xi_{0}$, and $\left.\operatorname{Hess}_{g}(u)\right|_{p}=H_{0}$.

Proof It is enough to do the proof in the case where $p \in M^{\text {int }}$. For if this has been done, and if $p \in \Gamma$, we may extend the manifold $M$ near $p$ to a slightly larger manifold $M_{1}$ so that $p \in M_{1}^{\text {int }}$ and $\partial M \backslash \Gamma \subset \subset \partial M_{1}$. Since $p$ is an interior point of $M_{1}$, we can find a harmonic function $u_{1}$ in $M_{1}$ having the correct second order Taylor expansion at $p$ and satisfying $\left.u_{1}\right|_{\partial M \backslash \Gamma}=0$. Choosing $f=\left.u_{1}\right|_{\partial M}$ implies that the solution of $-\Delta_{g} u=0$ in $M$ with $\left.u\right|_{\partial M}=f$ satisfies $u=\left.u_{1}\right|_{M}$ and has the required behaviour at $p$.

Thus, assume that $p \in M^{\text {int }}$. The proof will be given in four steps.

Step 1. First we find a local $g(p)$-harmonic function with prescribed 2-jet at $p$. Let $x=\left(x^{1}, \ldots, x^{n}\right)$ be normal coordinates in a small geodesic ball $U$ centered at $p$. In these coordinates, let $\xi_{0}=\left.\left(\xi_{0}\right)_{j} d x^{j}\right|_{p}$ and $H_{0}=\left.H_{j k} d x^{j} \otimes d x^{k}\right|_{p}$. Define the function

$$
u_{0}: U \rightarrow \mathbb{R}, \quad u_{0}\left(\exp _{p}\left(\left.x^{j} \partial_{j}\right|_{p}\right)\right)=a_{0}+\left(\xi_{0}\right)_{j} x^{j}+\frac{1}{2} H_{j k} x^{j} x^{k}
$$

Clearly $u_{0} \in C^{\infty}(\bar{U}), u_{0}(p)=a_{0}$ and $d u_{0}(p)=\xi_{0}$. The Hessian, computed in normal coordinates, is given by

$$
\left.\operatorname{Hess}_{g}\left(u_{0}\right)\right|_{p}=\left.\left(\partial_{x_{j} x_{k}} u_{0}-\Gamma_{j k}^{l} \partial_{x_{l}} u_{0}\right) d x^{j} \otimes d x^{k}\right|_{p}=H_{0}
$$

since $\left.\Gamma_{j k}^{l}\right|_{p}=0$ in normal coordinates. Since $H_{0}$ is trace free it follows that $\Delta_{g(p)} u_{0}=$ $\sum_{j=1}^{n} H_{j j}=0$ in $U$, i.e. $u_{0}$ is harmonic in $U$ with respect to the metric $g(p)$ with coefficients frozen at $p$.

Step 2. Next we find a local $g$-harmonic function near $p$ with 2-jet $\left(0,0, H_{0}\right)$. This is done by perturbing the functions $u_{0}$ from Step 1 in small balls, see e.g. [38, Proposition 5.10.4]. Let $u \in C^{\infty}(\overline{B(p, \varepsilon)})$ solve

$$
\Delta_{g} u=0 \text { in } B(p, \varepsilon),\left.\quad u\right|_{\partial B(p, \varepsilon)}=u_{0}
$$


where $u_{0}(x)=\frac{1}{2} H_{j k} x^{j} x^{k}$ in normal coordinates. We may rescale $\tilde{x}=x / \varepsilon, \tilde{u}(\tilde{x})=$ $\varepsilon^{-2} u(\varepsilon \tilde{x})$, so that $\tilde{u}$ solves (with derivatives taken with respect to $\tilde{x}$ )

$$
\left(g^{j k}(\varepsilon \tilde{x}) \partial_{j k}+\varepsilon \Gamma^{l}(\varepsilon \tilde{x}) \partial_{l}\right) \tilde{u}=0 \text { in } B_{1},\left.\quad \tilde{u}\right|_{\partial B_{1}}=\left.\tilde{u}_{0}\right|_{\partial B_{1}}=\frac{1}{2} H_{j k} \tilde{x}^{j} \tilde{x}^{k}
$$

Writing $\tilde{u}=\tilde{u}_{0}+\tilde{w}$ where $\tilde{u}_{0}(\tilde{x})=\varepsilon^{-2} u_{0}(\varepsilon \tilde{x})=u_{0}(\tilde{x})$, and using that $g^{j k}(0) \partial_{j k} \tilde{u}_{0}=0$, we see that $\tilde{w}$ solves

$$
\left(g^{j k}(\varepsilon \tilde{x}) \partial_{j k}+\varepsilon \Gamma^{l}(\varepsilon \tilde{x}) \partial_{l}\right) \tilde{w}=\tilde{F} \text { in } B_{1},\left.\quad \tilde{w}\right|_{\partial B_{1}}=0
$$

where $\|\tilde{F}\|_{H^{n / 2+3}\left(B_{1}\right)} \leq C \varepsilon$ since $g$ is smooth. By elliptic regularity (where the constants are uniform with respect to $0<\varepsilon<1$ ) and Sobolev embedding, $\|\tilde{w}\|_{C^{2}\left(\overline{B_{1}}\right)} \leq C \varepsilon$ where $C$ is uniform over small $\varepsilon$. Then also $\left\|u-u_{0}\right\|_{C^{2}(\overline{B(p, \varepsilon)})} \leq C \varepsilon$, which shows that there are local harmonic functions near $p$ with 2-jet arbitrarily close to $\left(0,0, H_{0}\right)$ at $p$.

We can make the 2 -jet at $p$ exactly equal to $\left(0,0, H_{0}\right)$ as follows. Consider the operator

$$
\begin{aligned}
& S:\{\text { local harmonic functions near } p\} \rightarrow \mathbb{R} \times T_{p}^{*} M \times\left(S_{\mathrm{tf}}^{2}\right)_{p} M, \\
& S u=\left(u(p), d u(p),\left.\operatorname{Hess}_{g}(u)\right|_{p}\right)
\end{aligned}
$$

where $\left(S_{\mathrm{tf}}^{2}\right)_{p} M$ is the space of trace free symmetric 2-tensors at $p$. The range of $S$ is a linear subspace of the finite-dimensional space $\mathbb{R} \times T_{p}^{*} M \times\left(S_{\mathrm{tf}}^{2}\right)_{p} M$, hence $\operatorname{Ran}(S)$ is closed. For any $H_{0} \in\left(S_{\mathrm{tf}}^{2}\right)_{p} M$ one has $\left(0,0, H_{0}\right) \in \overline{\operatorname{Ran}(S)}$, and thus there is a local harmonic function near $p$ with 2-jet $\left(0,0, H_{0}\right)$.

Step 3. We will next find a local $g$-harmonic function near $p$ with 2-jet $\left(a_{0}, \xi_{0}, H_{0}\right)$. In fact, the argument in Step 2 with the choice $u_{0}(x)=\left(\xi_{0}\right)_{j} x^{j}$ and scaling $\tilde{u}(\tilde{x})=$ $\varepsilon^{-1} u(\varepsilon \tilde{x})$ leads to local harmonic functions with 1 -jet at $p$ first arbitrarily close to $\left(0, \xi_{0}\right)$, and then exactly equal to $\left(0, \xi_{0}\right)$ as in the end of Step 2 . Adding one of the functions obtained in Step 2 yields a local harmonic function with 2-jet $\left(0, \xi_{0}, H_{0}\right)$, and adding a constant gives the 2-jet $\left(a_{0}, \xi_{0}, H_{0}\right)$.

Step 4. Finally, to find a global harmonic function with prescribed 2-jet at $p$, consider the operator

$$
T: C_{c}^{\infty}(\Gamma) \rightarrow \mathbb{R} \times T_{p}^{*} M \times\left(S_{\mathrm{tf}}^{2}\right)_{p} M, \quad f \mapsto\left(u_{f}(p), d u_{f}(p),\left.\operatorname{Hess}_{g}\left(u_{f}\right)\right|_{p}\right)
$$

where $u_{f}$ is the harmonic function in $M$ with $\left.u\right|_{\partial M}=f$. Given any $\left(a_{0}, \xi_{0}, H_{0}\right) \in$ $\mathbb{R} \times T_{p}^{*} M \times\left(S_{\mathrm{tf}}^{2}\right)_{p} M$, Step 3 shows that there is a local harmonic function $u_{0}$ in a small geodesic ball $U=B_{\varepsilon}(p)$ having 2-jet $\left(a_{0}, \xi_{0}, H_{0}\right)$ at $p$. Now Proposition A.2 implies that there is a sequence $\left(f_{j}\right) \subset C_{c}^{\infty}(\Gamma)$ such that $\left.u_{f_{j}}\right|_{U} \rightarrow u_{0}$ in $H^{n / 2+3}(U)$, hence $u_{f_{j}} \rightarrow u_{0}$ in $C^{2}(\bar{U})$ by Sobolev embedding. This shows that $T\left(C_{c}^{\infty}(\Gamma)\right)$ is a dense subspace of $\mathbb{R} \times T_{p}^{*} M \times\left(S_{\mathrm{tf}}^{2}\right)_{p} M$, but since the last space is finite dimensional $T$ has to be surjective. 
We can now prove the consequence stated in the beginning of the section:

Proof of Proposition A.1 (a) First assume that both $x$ and $y$ are in $M^{\text {int }}$. Let $U=B_{1} \cup B_{2}$ where $B_{1}$ and $B_{2}$ are balls centered at $x$ and $y$, which are chosen in such a way that $U \subset \subset M^{\text {int }}$ and $M \backslash \bar{U}$ is connected. Consider the harmonic function $u_{0}$ in $U$ with $\left.u_{0}\right|_{B_{1}}=1$ and $\left.u_{0}\right|_{B_{2}}=0$. By Proposition A.2, there exist $f_{j} \in C_{c}^{\infty}(\Gamma)$ such that

$$
\left\|\left.u_{f_{j}}\right|_{U}-u_{0}\right\|_{H^{n / 2+1}(U)} \rightarrow 0 \text { as } j \rightarrow \infty .
$$

By Sobolev embedding we also have $\left\|\left.u_{f_{j}}\right|_{U}-u_{0}\right\|_{L^{\infty}(U)} \rightarrow 0$. Choosing $f=f_{j}$ for large enough $j$ implies that $u_{f}(x) \neq u_{f}(y)$.

Now assume that $x \in M^{\text {int }}$ and $y \in \partial M$. We choose $\Gamma^{\prime} \subset \subset \Gamma \backslash\{y\}$, and use the argument above to find $f \in C_{c}^{\infty}\left(\Gamma^{\prime}\right)$ with $u_{f}(x) \neq 0$. Then one also has $u_{f}(x) \neq$ $u_{f}(y)=0$. Next, the case where $x \in \Gamma$ and $y \in M^{\text {int }}$ reduces to the previous case by interchanging $x$ and $y$. Finally, if $x \in \Gamma$ and $y \in \partial M$ with $x \neq y$, choose some $f \in C_{c}^{\infty}(\Gamma)$ with $f(x) \neq f(y)$ to obtain that $u_{f}(x) \neq u_{f}(y)$ as required.

(b) The result follows from Proposition A.5.

Finally, let us give a Runge approximation result for a linear source problem used for studying the inverse problem for nonlinear equations.

Proposition A.6 Let $(M, g)$ be a compact manifold with boundary and let $s \geq 1$. Let $W$ be an open subset of $M$, and let $U \subset \subset M^{\text {int }}$ be a domain with $C^{\infty}$ boundary such that $M \backslash \bar{U}$ is connected and $\bar{W} \cap \bar{U}=\emptyset$. Let also L be a second order uniformly elliptic differential operator on $M$ which is injective on $C^{\infty}(M) \cap H_{0}^{1}(M)$, and let $K: C_{c}^{\infty}(W) \rightarrow C^{\infty}(M), f \mapsto u$ be the solution operator for the problem

$$
L u=f \text { in } M,\left.\quad u\right|_{\partial M}=0 .
$$

Then the set

$$
\mathcal{R}=\left\{\left.K f\right|_{U} ; f \in C_{c}^{\infty}(W)\right\}
$$

is dense in the space $\mathcal{S}=\left\{u \in H^{s}(U): L u=0\right.$ in $\left.U\right\}$ with respect to the $H^{s}(U)$ norm.

Proof Let $F \in\left(H^{s}(U)\right)^{*}$ satisfy $F\left(\left.K f\right|_{U}\right)=0$ for all $f \in C_{c}^{\infty}(W)$. By the HahnBanach theorem, it is enough to show that $F(u)=0$ for all $u \in \mathcal{S}$. As in the proof of Proposition A.2, we take $(M, g) \subset \subset\left(M_{1}, g\right) \subset \subset(N, g)$ and extend $L$ to an elliptic second order operator $A$ on $N$. Given $F \in\left(H^{S}(U)\right)^{*}$, we define $\hat{F} \in H^{-s}(N)$, $\operatorname{supp}(\hat{F}) \subset \bar{U}$, by

$$
\hat{F}(w)=F\left(\left.w\right|_{U}\right), \quad w \in H^{s}(N) .
$$

Continuing as in the proof of Proposition A.2, we may find $\tilde{F} \in H^{-s}(N)$ so that

$$
\tilde{F}=\hat{F}+\eta
$$


with $\operatorname{supp}(\eta) \subset V$, where $V \subset N \backslash M_{1}$, and $\tilde{F}$ is $L^{2}$-orthogonal to the kernel of $A$. Moreover we may find $\tilde{F}_{j} \in C_{c}^{\infty}\left(M^{\text {int }} \cup V\right)$ with $\tilde{F}_{j} \rightarrow \tilde{F}$ in $H^{-s}(N)$, and using the same procedure as in the proof of Proposition A.2 we can represent $\tilde{F}$ and $\tilde{F}_{j}$ as

$$
A^{*} v=\tilde{F} \text { in } N, \quad A^{*} v_{j}=\tilde{F}_{j} \text { in } N .
$$

Similarly, in $M$ we have

$$
\begin{aligned}
& L^{*} \varphi_{j}=\tilde{F}_{j} \text { in } M^{\text {int }},\left.\quad \varphi_{j}\right|_{\partial M}=0, \\
& L^{*} \varphi=\tilde{F} \text { in } M^{\text {int }},\left.\quad \varphi\right|_{\partial M}=0,
\end{aligned}
$$

where $\varphi_{j} \in C^{\infty}(M), \varphi \in H^{-s+2}(M)$, and $\varphi$ is smooth near $\partial M$.

Let $E$ be a bounded extension operator $H^{s}(M) \rightarrow H^{s}(N)$ satisfying $\operatorname{supp}(E w) \subset$ $M_{1}^{\text {int }}$ for all $w \in H^{s}(M)$. Since $F\left(\left.K f\right|_{U}\right)=0$ for $f \in C_{c}^{\infty}(W)$, we have

$$
\begin{aligned}
0 & =F\left(\left.K f\right|_{U}\right)=\tilde{F}(E(K f))=\lim \tilde{F}_{j}(E(K f)) \\
& =\lim \left(A^{*} v_{j}, E(K f)\right)_{N} .
\end{aligned}
$$

In the last expression, $E(K f)$ is supported in $M_{1}^{\text {int }}$ and $\left.A^{*} v_{j}\right|_{M_{1}}$ vanishes outside a neighborhood of $\bar{U} \subset M^{\text {int }}$. Thus this expression can be understood as an integral over $M$. Integrating by parts, we get

$$
0=\lim \left(L^{*} \varphi_{j}, K f\right)_{M}=\lim \left(\varphi_{j}, L K f\right)_{M}=\lim \left(\varphi_{j}, f\right)_{W}
$$

Here we used that $K f$ and $\varphi_{j}$ vanish on $\partial M$ and thus the boundary terms vanish. The higher regularity estimate in Lemma A.3 implies that $\varphi_{j} \rightarrow \varphi$ in $H^{m}(W)$ for any $m \geq 0$, and consequently

$$
0=(\varphi, f)_{W}
$$

Since the previous result holds for all $f \in C_{c}^{\infty}(W)$, we see that $\varphi$ solves

$$
L^{*} \varphi=0 \text { in } M^{\text {int }} \backslash \bar{U},\left.\quad \varphi\right|_{W} \equiv 0 .
$$

Also, $\varphi$ is smooth in the connected set $M \backslash \bar{U}$. The unique continuation principle implies that $\left.\varphi\right|_{M \backslash \bar{U}}=0$. Thus $\varphi \in H_{\bar{U}}^{-s+2}(N)$. As in the proof of Proposition A.2, we have $\psi_{j} \in C_{c}^{\infty}(U), \psi_{j} \rightarrow \varphi$ in $H^{-s+2}(N)$. If $E$ denotes a bounded extension operator from $H^{s}(U)$ to $H^{s}(N)$ with $\operatorname{supp}(E w) \subset M^{\text {int }}$ for any $w \in H^{s}(U)$, we have

$$
F(u)=\tilde{F}(E u)=\left(A^{*} v, E u\right)_{N}=\lim \left(A^{*} \psi_{j}, E u\right)_{N}=\lim \left(L^{*} \psi_{j}, u\right)_{U} .
$$

If $u \in \mathcal{S}$, we can integrate by parts to see that $F(u)=0$.

As a consequence, we obtain an analogue of Proposition A.1 where boundary sources are replaced by interior sources. 
Proposition A.7 Let $(M, g)$ be a compact manifold with boundary, and let L be a second order uniformly elliptic differential operator on $M$ which is injective on $C^{\infty}(M) \cap H_{0}^{1}(M)$. Let $W$ be a nonempty open subset of $M$, and denote by $u_{f}$ the solution of $L u=f$ in $M$ with $\left.u\right|_{\partial M}=0$.

(a) If $x \in M^{\text {int },} y \in M$ and $x \neq y$, there is $f \in C_{c}^{\infty}(W)$ such that

$$
u_{f}(x) \neq u_{f}(y)
$$

(b) If $x \in M^{\text {int }}$ and $v \in T_{x}^{*} M$, there is $f \in C_{c}^{\infty}(W)$ such that

$$
d u_{f}(x)=v .
$$

Proof The proof is analogous to that of Proposition A.1, except that we need a version of Proposition A.5 for 0 - and 1 -jets where $-\Delta_{g}$ is replaced by $L$ (this can be found in [3, Theorem I.5.4.1]), and we need to use the Runge approximation result in Proposition A.6.

\section{References}

1. Baird, P., Wood, J.C.: Harmonic morphisms between Riemannian manifolds. In: London Mathematical Society Monographs. New Series, vol. 29. The Clarendon Press, Oxford University Press, Oxford (2003)

2. Belishev, M.: Geometrization of rings as a method for solving inverse problems. In: Sobolev Spaces in Mathematics. III, Int. Math. Ser. (N.Y.), vol. 10. Springer, New York, pp. 5-24 (2009)

3. Bers, L., John, F., Schechter, M.: Partial Differential Equations. American Mathematical Society, Providence (1979)

4. Browder, F.E.: Approximation by solutions of partial differential equations. Am. J. Math. 84, 134-160 (1962)

5. Cekić, M.: A contribution to the Calderón problem for Yang-Mills connections. arXiv:1704.01362

6. Chandler-Wilde, S.N., Hewett, D.P., Moiola, A.: Sobolev spaces on non-Lipschitz subsets of $R^{n}$ with application to boundary integral equations on fractal screens. Integral Equ. Oper. Theory 87, 179-224 (2017)

7. Daudé, T., Kamran, N., Nicoleau, F.: On the hidden mechanism behind non-uniqueness for the anisotropic Calderón problem with data on disjoint sets. arXiv:1701.09056

8. Deng, Y., Liu, H., Uhlmann, G.: Full and partial cloaking in electromagnetic scattering. Arch. Ration. Mech. Anal. 223, 265-299 (2017)

9. Deturck, D., Kazdan, J.: Some regularity theorems in Riemannian geometry. Annales de l'ENS 14, 249-260 (1981)

10. Dos Santos Ferreira, D., Kenig, C.E., Salo, M., Uhlmann, G.: Limiting Carleman weights and anisotropic inverse problems. Invent. Math. 178, 119-171 (2009)

11. Dos Santos Ferreira, D., Kurylev, Y., Lassas, M., Liimatainen, T., Salo, M.: The linearized Calderón problem in transversally anisotropic geometries. Int. Math. Res. Not. (2018). https://doi.org/10.1093/ imrn/rny234

12. Dos Santos Ferreira, D., Kurylev, Y., Lassas, M., Salo, M.: The Calderón problem in transversally anisotropic geometries. JEMS 18, 2579-2626 (2016)

13. Fuglede, B.: Harmonic morphisms between Riemannian manifolds. Ann. Inst. Fourier (Grenoble) 28(2), 107-144 (1978)

14. Gilbarg, D., Trudinger, N.S.: Elliptic Partial Differential Equations of Second Order. Springer, Berlin (1998)

15. Greenleaf, A., Kurylev, Y., Lassas, M., Uhlmann, G.: Full-wave invisibility of active devices at all frequencies. Commun. Math. Phys. 275, 749-789 (2007) 
16. Greenleaf, A., Kurylev, Y., Lassas, M., Uhlmann, G.: Cloaking devices, electromagnetic wormholes and singular transformation optics. SIAM Rev. 51, 3-33 (2009)

17. Greenleaf, A., Kurylev, Y., Lassas, M., Uhlmann, G.: Invisibility and inverse problems. Bull. AMS 46, 55-97 (2009)

18. Greenleaf, A., Lassas, M., Uhlmann, G.: On nonuniqueness for Calderón's inverse problem. Math. Res. Lett. 10, 685-693 (2003)

19. Greene, R.E., Wu, H.: Embedding of open Riemannian manifolds by harmonic functions. Ann. Inst. Fourier (Grenoble) 25(1), 215-235 (1975)

20. Guillarmou, C., Sá Barreto, A.: Inverse problems for Einstein manifolds. Inverse Probl. Imaging 3, 1-15 (2009)

21. Guillarmou, C., Salo, M., Tzou, L.: The linearized Calderón problem on complex manifolds. Acta Math. Sinica (2018). arXiv:1805.00752 (to appear)

22. Hervas, D., Sun, Z.: An inverse boundary value problem for quasilinear elliptic equations. Commun. PDE 27, 2449-2490 (2002)

23. Hörmander, L.: The Analysis of Linear Partial Differential Operators, vol. III. Springer, Berlin (1985)

24. Isakov, V.: On uniqueness in inverse problems for semilinear parabolic equations. Arch. Rat. Mech. Anal. 124, 1-12 (1993)

25. Isakov, V.: Inverse Problems for Partial Differential Equations, 2nd edn. Springer, Berlin (2006)

26. Lassas, M., Liimatainen, T., Salo, M.: The Calderón problem for the conformal Laplacian. arXiv: 1612.07939

27. Lassas, M., Taylor, M., Uhlmann, G.: The Dirichlet-to-Neumann map for complete Riemannian manifolds with boundary. Commun. Anal. Geom. 11, 207-222 (2003)

28. Lassas, M., Uhlmann, G.: Determining a Riemannian manifold from boundary measurements. Annales de l'ENS 34(5), 771-787 (2001)

29. Lax, P.D.: A stability theorem for solutions of abstract differential equations, and its application to the study of the local behavior of solutions of elliptic equations. Commun. Pure Appl. Math. 9(4), 747-766 (1956)

30. Lee, J.M., Uhlmann, G.: Determining anisotropic real-analytic conductivities by boundary measurements. Commun. Pure Appl. Math. 42, 1097-1112 (1989)

31. Malgrange, B.: Existence et approximation des solutions des équations aux dérivées partielles et des équations de convolution. Ann. Inst. Fourier Grenoble, vol. 6, pp. 271-355 (1955-1956)

32. McLean, W.: Strongly Elliptic Systems and Boundary Integral Equations. Cambridge University Press, Cambridge (2000)

33. Morrey, Charles B.: On the analyticity of the solutions of analytic non-linear elliptic systems of partial differential equations: part I. Analyticity in the interior. Am. J. Math. 80(1), 198-218 (1958)

34. Rüland, A., Salo, M.: Quantitative Runge approximation and inverse problems. IMRN. arXiv:1708.06307 (to appear)

35. Salo, M., Zhong, X.: An inverse problem for the $p$-Laplacian: boundary determination. SIAM J. Math. Anal. 44, 2474-2495 (2012)

36. Sun, Z.: Conjectures in inverse boundary value problems for quasilinear elliptic equations. Cubo 7(3), 65-74 (2005)

37. Sun, Z., Uhlmann, G.: Inverse problems in quasilinear anisotropic media. Am. J. Math. 119, 771-797 (1997)

38. Taylor, M.E.: Partial Differential Equations, vol. I. Springer, Berlin (1996)

39. Taylor, M.E.: Existence and regularity of isometries. Trans. Am. Math. Soc. 358, 2414-2423 (2006)

Publisher's Note Springer Nature remains neutral with regard to jurisdictional claims in published maps and institutional affiliations. 